\title{
Cherenkov radiation of a charge exiting open-ended waveguide with dielectric filling
}

\author{
Sergey N. Galyamin, Andrey V. Tyukhtin, ${ }^{\dagger}$ Viktor V. Vorobev, and Alexandra A. Grigoreva \\ Saint Petersburg State University, 7/9 Universitetskaya naberezhnaya, St. Petersburg 199034 Russia \\ Alexander S. Aryshev \\ KEK: High Energy Accelerator Research Organization, 1-1 Oho, Tsukuba, Ibaraki 305-0801 Japan
}

(Received 25 June 2018; published 2 January 2019)

\begin{abstract}
We consider a semi-infinite open-ended cylindrical waveguide with uniform dielectric filling placed into collinear infinite vacuum waveguide with larger radius. Electromagnetic field produced by a point charge or Gaussian bunch moving along structure's axis from the dielectric waveguide into the vacuum one is investigated. We utilize the modified residue-calculus technique and obtain rigorous analytical solution of the problem by determining coefficients of mode excitation in each subarea of the structure. The main attention is paid to analysis of penetration of Cherenkov radiation into vacuum regions of the outer waveguide. Numerical simulations in CST Particle Studio are also performed (for long enough bunch exciting the first Cherenkov mode only) and an excellent agreement between analytical and simulated results is shown. The discussed structure can be used for generation of Terahertz radiation by modulated bunches (bunch trains) by means of high-order Cherenkov modes. In this case, due to high frequencies numerical simulations become extremely difficult while the developed analytical technique still remains the efficient approach for calculation of the radiation characteristics.
\end{abstract}

DOI: $10.1103 /$ PhysRevAccelBeams.22.012801

\section{INTRODUCTION}

In recent years, an essential interest is observed in the area of contemporary sources of Terahertz $(\mathrm{THz})$ radiation based on beam-driven waveguide structures loaded with dielectric. Despite of the fact that both ordinary vacuum $\mathrm{THz}$ devices (such as classical backward wave oscillator) are widely available and other mechanisms for $\mathrm{THz}$ sources are discussed (see, e.g., Refs. [1-4]), beam-driven sources are still extremely attractive due to extraordinary $\mathrm{THz}$ radiation peak power [5]. According to this idea, Cherenkov radiation should be generated by wellcontrolled electron bunch passed through a waveguide structure with dielectric filling and open aperture [6,7]. The electron bunch should be modulated so that a highorder Cherenkov frequency is excited, therefore allowing the use of, for example, mm-sized waveguides for $\mathrm{THz}$ generation. Another challenge here is efficient extraction of the radiation from inside the structure into free space. The possibilities for using the nonorthogonal end cut for this

\footnotetext{
*s.galyamin@spbu.ru

a.tyuhtin@spbu.ru
}

Published by the American Physical Society under the terms of the Creative Commons Attribution 4.0 International license. Further distribution of this work must maintain attribution to the author(s) and the published article's title, journal citation, and DOI. purpose were theoretically estimated [8] and experimentally confirmed $[9,10]$. Nevertheless, rigorous solution for the electromagnetic (EM) field produced by a charged particle bunch passing from the open-ended circular waveguide with dielectric filling is still missing even in the case of orthogonal end cut. In particular, such a solution is required for determination of the area of applicability of the approximate technique used in [8] and it's possible improvement.

General theory for analysis of radiation from open-ended waveguide structures was actively developed during several preceding decades [11-13]. Typically, the theory of EM processes for the discussed waveguide discontinuity (open end) was constructed for vacuum case and excitation by single waveguide mode, however, vacuum structures excited by a moving charge were also partially investigated [14-18]. It should be also noted here that analytical approaches becomes essentially more complicated while they deal with the structures containing dielectric inclusions $[12,19,20]$.

In a series of recent papers, we started rigorous investigation of the aforementioned problem on EM field in a circular open-ended waveguide with orthogonal cut and dielectric filling excited by the field of a moving charged particle bunch [21-25]. In these publications, the semiinfinite waveguide was placed (embedded) into collinear infinite vacuum waveguide with larger radius. Therefore the considered structure is further referred to as "embedded" 
structure. Based on a very good agreement between analytical and simulated (using both COMSOL and CST) results observed in the aforementioned papers one can conclude that convenient analytical technique for solution of the described problem was fully approved.

However, Cherenkov radiation which is responsible for the aforementioned high-power $\mathrm{THz}$ emission and therefore is of most interest in the structure under consideration was not described in details. In the present paper, we give the detailed analytical solution for EM field generated by a charged particle bunch in the "embedded" structure loaded with dielectric. We apply the modified residue-calculus technique [19] and describe penetration of Cherenkov radiation into vacuum regions of the structure (this fact explains the term "Cherenkov radiation" in the title). In the case under consideration, the process of penetration occurs due to the diffraction mechanism.

It should be noted that the considered closed geometry (the "embedded" geometry) has several advantages compared to the opened one for theory, simulations and possible experiments. First, closed structure possesses discrete mode spectrum, thus simplifying analytical consideration. Second, finite area of EM field existence allows efficient simulations without the need of extremely large amount of computational resources.

Third, various vacuum "embedded" structures have been investigated previously for a number of applications. For example, one could mention extensive studies of various inhomogeneities of the "liner" (the inner metallic pipe shielding the outer cooled pipe from the parasitic heating of synchrotron radiation) of the high current storage rings of the superconducting colliders [26-42]. Some of these structures are very close in geometry to the structure discussed in the present paper (in the case without dielectric loading), for example, [32,36,37]. Also similar vacuum structures containing coaxial areas were considered in the context of bunch diagnostic system developments (especially for a Cherenkov-based bunch position monitors) [43-45] or efficient linac collimators [46]. A recent trend for luminosity upgrade of LHC has resulted in investigation of "crab cavities" $[47,48]$ which are also partially related to discussed structures with coaxial areas attached to the main circular waveguide where a bunch propagates. Impedance of a finite length cavity with dielectric filling are also investigated [49].

Therefore, practical realization of the discussed "embedded" structure does not lead to a principal limitations. Moreover, at the contemporary accelerators, experiments on bunch radiation are typically conducted in a vacuum chamber. The outer waveguide of our structure can be a convenient model description of the chamber walls in the case of collinear cylindrical chamber and large enough radius of the outer waveguide.

The paper is organized as follows. After the Introduction (Sec. I), we present rigorous solution of the problem
(Sec. II). Note that this section contains only final analytical results while details of calculations and intermediate derivations are placed into three appendices (Appendices A, B and C) succeeding the main text. Section III presents numerical results visualizing the obtained rigorous formulas, simulated results (via CST PS package) and comparison between them. Section IV contains conclusions and discussions and finishes the paper.

\section{ANALYTICAL RESULTS}

Geometry of the problem under consideration is shown in Fig. 1. A semi-infinite perfectly conducting circular waveguide with radius $b$ filled with a homogeneous dielectric $(\varepsilon>1)$ is put into a concentric infinite waveguide with radius $a>b$. The structure is excited by a point charge $q$ moving along $z$-axis with constant velocity $\vec{V}=$ $V \vec{e}_{z}=\beta c \vec{e}_{z}$ ( $c$ is the light speed in vacuum). Comments on limitations of the given model having no channel for a charge passage through the dielectric will be given in Sec. IV. Corresponding charge density $\rho$ and current density $\vec{j}=j \vec{e}_{z}$ have the form

$$
\rho=q \delta(x) \delta(y) \delta(z-V t), \quad j=V \rho .
$$

Unless otherwise specified, analytical results presented below correspond to the source (1). These results can be easily generalized for the case of a bunch being infinitesimally thin in $x y$-plane, similar to (1), but having arbitrary charge distribution $\eta(z-V t)$ along $z$ (longitudinal) direction. In this case, charge and current densities, $\rho_{b}$ and $\vec{j}_{b}=j_{b} \vec{e}_{z}$ are:

$$
\rho_{b}=q \delta(x) \delta(y) \eta(z-V t), \quad j_{b}=V \rho_{b} .
$$

As can be easily shown, to obtain formulas related to the case of the bunch (2) one should substitute

$$
q \rightarrow 2 \pi q \tilde{\eta}(\omega / V)
$$

where $\tilde{\eta}(\omega / V)$ is the Fourier transform

$$
\tilde{\eta}(\xi)=(2 \pi)^{-1} \int_{-\infty}^{+\infty} \eta(\zeta) e^{-i \xi \zeta} d \zeta
$$

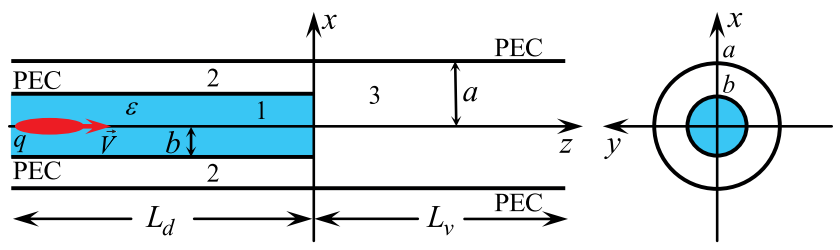

FIG. 1. Geometry of the problem and main notations. $L_{v}$ and $L_{d}$ are lengths of vacuum and dielectric parts of the model, correspondingly. They are infinite in theory and finite in simulations. 
calculated for $\xi=\omega / V$. For example, in the case of Gaussian bunch with the rms half-length $\sigma$,

$$
\eta_{\mathrm{G}}(z-V t)=\frac{1}{\sqrt{2 \pi} \sigma} \exp \left(\frac{-(z-V t)^{2}}{2 \sigma^{2}}\right),
$$

and one should substitute

$$
q \rightarrow q \exp \left(-\frac{\omega^{2}}{\omega_{\sigma}^{2}}\right), \quad \omega_{\sigma}=\frac{\sqrt{2} V}{\sigma} .
$$

In this case, the largest essential frequency in the spectrum $\omega_{\max }$ is determined so that Gaussian exponential term in (6) results in certain predetermined attenuation for $\omega=\omega_{\max }$. Typical attenuation (for example, used in CST PS code by default) is $-20 \mathrm{~dB}$, that is

$$
20 \lg \left[1 / \exp \left(-\omega_{\max }^{2} / \omega_{\sigma}^{2}\right)\right]=-20 .
$$

This result in $\omega_{\max }=\omega_{\sigma} \sqrt{\ln 10} \approx 1.5 \omega_{\sigma}$.

In principle, the problem formulation can be extended to a more realistic case of a three-dimensional bunch. Simplest generalization can be done for bunches being symmetrical with respect to $z$-axis, in this case the incident field (Sec. II A) should be multiplied by the corresponding form-factor [50], while the scattered field modal expansion (Sec. II B) becomes the same since only symmetrical ( $\phi$-independent) modes are excited. Consideration of asymmetrical bunches is rather more complicated since both the incident field and decomposition for the scattered field should be reexamined essentially.

Further the cylindrical frame $r, \phi, z$ (associated with the Cartesian frame shown in Fig. 1) is used. The problem will be solved in the frequency domain, so that Fourier harmonic $H_{\omega \phi}$ will be determined. Other nonzero field components are calculated as follows:

$$
\begin{gathered}
E_{\omega r}=c(i \omega \varepsilon)^{-1} \partial H_{\omega \phi} / \partial z, \\
E_{\omega z}=-c(i \omega \varepsilon r)^{-1}\left[H_{\omega \phi}+r \partial H_{\omega \phi} / \partial r\right] .
\end{gathered}
$$

Time-domain field dependencies are calculated using the inverse Fourier transform formulas which can be transformed to the following form [51,52]:

$$
H_{\phi}(r, z, t)=2 \operatorname{Re} \int_{0}^{+\infty} H_{\omega \phi} e^{-i \omega t} d \omega .
$$

On the basis of Eq. (10), it is sufficient to consider only positive frequencies in the spectrum.

\section{A. Incident field}

Fourier harmonic of the magnetic component of the incident field has the following form [53]:

$$
H_{\omega \phi}^{(i)}= \begin{cases}H_{\omega \phi}^{(i 1)}, & \text { for } z<0 \\ H_{\omega \phi}^{(i 3)}, & \text { for } z>0 .\end{cases}
$$

Here

$$
\begin{gathered}
H_{\omega \phi}^{(i 1)}=\frac{i q s}{2 c}\left[H_{1}^{(1)}(r s)-\frac{H_{0}^{(1)}(b s)}{J_{0}(b s)} J_{1}(r s)\right] e^{\frac{i \omega z}{V},} \\
s(\omega)=\sqrt{\omega^{2} V^{-2}\left(\varepsilon \beta^{2}-1\right)}, \operatorname{Im} s>0, \\
H_{\omega \phi}^{(i 3)}=\frac{i q s_{0}}{2 c}\left[H_{1}^{(1)}\left(r s_{0}\right)-\frac{H_{0}^{(1)}\left(a s_{0}\right)}{J_{0}\left(a s_{0}\right)} J_{1}\left(r s_{0}\right)\right] e^{\frac{i \omega z}{V}},
\end{gathered}
$$

$s_{0}(\omega)=\sqrt{\omega^{2} V^{-2}\left(\beta^{2}-1\right)}, \operatorname{Im} s_{0}>0, J_{0,1}$ are Bessel functions, $H_{0,1}^{(1)}$ are Hankel functions of the first order. Equation (12) represents the total field of a point charge (1) uniformly moving in regular waveguide of radius $b$ filled with dielectric $\varepsilon$. Equation (13) represents the total field of the same charge moving in regular vacuum waveguide with radius $a$.

For $\varepsilon \beta^{2}>1$, incident field in the inner dielectric waveguide $H_{\omega \phi}^{(i 1)}$ contains field of Cherenkov radiation (so called "wakefield"). Wakefield is the part of (12) with the discrete frequency spectrum, namely the finite set of real "Cherenkov frequencies" $\omega_{l}^{\mathrm{Ch}}$ which correspond to real positive poles of the expression (12). These poles are determined by the following equation:

$$
J_{0}(b s)=0 \Rightarrow s\left(\omega_{l}^{\mathrm{Ch}}\right)=j_{0 l} / b,
$$

where $j_{0 l}$ is the zero of the zero-order Bessel function, $J_{0}\left(j_{0 l}\right)=0, l=1,2, \ldots$ It can be shown (for example, by the limiting process from the case with dissipation taken into account in dielectric) that the integration path in (10) passes real "Cherenkov poles" from above. Therefore, these poles contribute to the incident field only behind the charge, i.e. for $\zeta=z-V t<0$. Contributions of these poles (residues) can be calculated:

$$
H_{\phi}^{\mathrm{Ch}(i 1)}(r, z, t)=\sum_{l=1}^{\infty} H_{\phi l}^{\mathrm{Ch}(i 1)}(r, z, t),
$$

where

$$
\begin{aligned}
H_{\phi l}^{\mathrm{Ch}(i 1)}(r, z, t) & =2 \operatorname{Re}\left[(-2 \pi i) \operatorname{Res}_{\omega_{l}^{\mathrm{Ch}}} H_{\omega \phi}^{(i 1)} e^{-i \omega_{l}^{\mathrm{Ch}} t}\right] \\
& =2 \operatorname{Re}\left[\frac{\pi q \omega_{l}^{\mathrm{Ch}}}{b c} \frac{H_{0}^{(1)}\left(j_{0 l}\right)}{J_{1}\left(j_{0 l}\right)} J_{1}\left(r j_{0 l} / b\right) e^{i \omega_{l}^{\mathrm{Ch}} \zeta / V}\right] .
\end{aligned}
$$

In the case of Gaussian bunch (5) due to vanishing exponential term (6) in the spectrum, high-order Cherenkov 
frequencies are strongly suppressed, therefore one can obtain monochromatic Cherenkov radiation for long enough bunch (this is also true for arbitrary finite length bunch).

In the geometry under consideration (see Fig. 1), the waveguide with dielectric filling has an open end, therefore Cherenkov radiation generated inside the inner waveguide will penetrate both coaxial part of the structure (area 2 in Fig. 1) and wide vacuum part (area 3 in in Fig. 1). Note that penetration of Cherenkov radiation through simple plane infinite interface between two media accompanying generation of transition radiation was investigated previously [52,54-56]. In the case under consideration, the process of penetration occurs due to the diffraction mechanism. This process is of main interest in this paper and it can be described by the theory presented below.

\section{B. Scattered field}

The unknown additional (scattered) field propagating from the boundary in the domains 1,2 and 3 can be presented as standard series over corresponding waveguide modes [12]:

$$
\begin{gathered}
H_{\omega \phi}^{(1)}(r, z)=\sum_{m=1}^{\infty} B_{m} J_{1}\left(r j_{0 m} / b\right) e^{\kappa_{z m}^{(1)} z}, \\
H_{\omega \phi}^{(3)}(r, z)=\sum_{m=1}^{\infty} A_{m} J_{1}\left(r j_{0 m} / a\right) e^{-\gamma_{z m}^{(3)} z}, \\
H_{\omega \phi}^{(2)}(r, z)=C_{0} r^{-1} e^{\gamma_{z 0}^{(2)} z}+\sum_{m=1}^{\infty} C_{m} Z_{m}\left(r \chi_{m}\right) e^{\gamma_{z m}^{(2)} z},
\end{gathered}
$$

where $\left\{A_{m}\right\},\left\{B_{m}\right\}$ and $\left\{C_{n}\right\}$ are unknown modal expansion coefficients [12]. Note that the first term in the righthand side of (19) represents the TEM wave with $E_{\omega z}=0$, in accordance with (9). Here

$$
Z_{m}(\xi)=J_{1}(\xi)-N_{1}(\xi) J_{0}\left(a \chi_{m}\right) N_{0}^{-1}\left(a \chi_{m}\right)
$$

is the transversal eigenfunction of the coaxial region (area 2 in Fig. 1), $\chi_{m}>0$ is the solution of the dispersion relation for the area 2 ,

$$
J_{0}\left(b \chi_{p}\right) N_{0}\left(a \chi_{p}\right)-J_{0}\left(a \chi_{p}\right) N_{0}\left(b \chi_{p}\right)=0,
$$

$N_{0}$ is the Neumann function. Propagation constants are

$$
\begin{gathered}
\kappa_{z m}^{(1)}=\sqrt{j_{0 m}^{2} b^{-2}-\varepsilon k_{0}^{2}}, \\
\gamma_{z m}^{(3)}=\sqrt{j_{0 m}^{2} a^{-2}-k_{0}^{2}},
\end{gathered}
$$

$$
\gamma_{z 0}^{(2)}=-i k_{0}, \quad \gamma_{z m}^{(2)}=\sqrt{\chi_{m}^{2}-k_{0}^{2}},
$$

where $k_{0}=\omega / c, \operatorname{Re} \kappa_{z m}^{(1)}>0, \operatorname{Re} \gamma_{z m}^{(2,3)}>0, m=1,2, \ldots$

For readers' convenience, below we discuss the way for solving the problem under consideration just briefly and present the main resulting formulas only. Rather cumbersome details of calculations needed for deep understanding of the used technique are moved into the Appendices. Corresponding references are given in the text.

Performing matching of the components $H_{\omega \phi}$ and $E_{\omega r}$ for $z=0$, and eliminating the $r$-dependence from the resulting relations, after certain analytical transformations we obtain infinite systems for unknown coefficients $\left\{A_{m}\right\}$, $\left\{B_{m}\right\}$ and $\left\{C_{n}\right\}(n=0,1,2, \ldots)$ of mode decompositions (18), (17), and (19), correspondingly. This procedure is explained in detail in Appendix A, where obtained systems (A10), (A11), (A18), and (A19) are presented. Using the modified residue-calculus technique [12,23], these systems can be solved by constructing specific complex-valued function $f(w)$. This procedure is described in detail in the Appendix B. Finally, the coefficients can be expressed through $f(w)$ as follows:

$$
\begin{gathered}
A_{m}=\frac{\operatorname{Res}_{\gamma_{z m}^{(3)}} f(w)}{J_{0}\left(b j_{0 m} / a\right) j_{0 m} / a}, \\
B_{m}=\frac{\varepsilon \gamma_{z m}^{(1)}+\kappa_{z m}^{(1)}}{2 b J_{1}\left(j_{0 m}\right) \gamma_{z m}^{(1)} \kappa_{z m}^{(1)}}\left[\frac{i q}{2 c b}\left(R_{m} F_{d m}^{+}+F_{d m}^{-}\right)\right. \\
\left.-R_{m} F_{v m}^{-}-F_{v m}^{+}-R_{m} f\left(\gamma_{z m}^{(1)}\right)-f\left(-\gamma_{z m}^{(1)}\right)\right], \\
C_{0}=\frac{f\left(-\gamma_{z 0}^{(2)}\right)}{2 \gamma_{z 0}^{(2)} \ln (a / b)}, \\
C_{m}=\frac{f\left(-\gamma_{z m}^{(2)}\right)}{2 \gamma_{z m}^{(2)}\left[\frac{a^{2} Z_{m}^{2}\left(a \chi_{m}\right)}{2 b Z_{m}\left(b \chi_{m}\right)}-\frac{b}{2} Z_{m}\left(b \chi_{m}\right)\right]} .
\end{gathered}
$$

Here

$$
\gamma_{z m}^{(1)}=\sqrt{j_{0 m}^{2} b^{-2}-k_{0}^{2}}, \quad \operatorname{Re} \gamma_{z m}^{(1)}>0
$$

is the propagation constant of the area 1 in the case of $\varepsilon=1$,

$$
\begin{gathered}
R_{m}=\frac{\varepsilon \gamma_{z m}^{(1)}-\kappa_{z m}^{(1)}}{\varepsilon \gamma_{z m}^{(1)}+\kappa_{z m}^{(1)}}, \\
F_{d m}^{ \pm}=\frac{2 i j_{0 p}}{\pi b} \frac{\frac{\omega}{i V \varepsilon} \pm \gamma_{z m}^{(1)}}{s^{2}-\left(j_{0 m} / b\right)^{2}},
\end{gathered}
$$




$$
F_{v m}^{ \pm}=\frac{2 i j_{0 m}(\pi b)^{-1}}{\frac{\omega}{i V} \pm \gamma_{z m}^{(1)}} .
$$

Function $f(w)$ is determined as follows:

$$
\begin{gathered}
f(w)=\frac{P g(w)}{w-\frac{\omega}{i V}}, \\
g(w)=\frac{\left(w-\gamma_{z 0}^{(2)}\right) \prod_{n=1}^{\infty}\left(1-\frac{w}{\gamma_{z n}^{(2)}}\right)}{\prod_{m=1}^{\infty}\left(1-\frac{w}{\gamma_{z m}^{(3)}}\right)} \prod_{s=1}^{\infty}\left(1-\frac{w}{\Gamma_{s}}\right) Q(w), \\
Q(w)=\exp \left[-\frac{w}{\pi}\left(b \ln \left(\frac{b}{a-b}\right)+a \ln \left(\frac{a-b}{a}\right)\right)\right], \\
P=\frac{i q}{2 c} \frac{i s_{0}^{2} h_{0}}{g(\omega /(i V))}, \\
h_{0}=N_{0}\left(b s_{0}\right)-N_{0}\left(a s_{0}\right) J_{0}\left(b s_{0}\right) / J_{0}\left(a s_{0}\right) .
\end{gathered}
$$

The correct construction of the function $f(w)$ (33) is the key point of the residue-calculus technique. The details of this procedure is described in detail in the Appendix B. In particular, one should determine zeros $\left\{\Gamma_{m}\right\}$ shifted with respect to zeros of vacuum problem $\left\{\gamma_{z m}^{(1)}\right\}$ :

$$
\Gamma_{m}=\gamma_{z m}^{(1)}+\frac{\pi}{b} \Delta_{m},
$$

where the set $\left\{\Delta_{m}\right\}$ determines the unknown shifts. Shifted zeros of $f(w)$ are the distinguishing feature of the problem with dielectric and this fact complicates significantly the solution (compared to the vacuum case) because the set $\left\{\Gamma_{m}\right\}$ is determined for each distinct frequency $\omega$. Since this is connected with iterative solution of a certain complicated nonlinear system [see Eq. (B14) in Appendix B], it is difficult to obtain the field spectrum $H_{\omega \phi}$ for significant range of frequencies $\left[0, \omega_{\max }\right]$. Therefore, it is difficult to calculate full time dependencies for the field components using inverse Fourier transform formulas (10).

However, the detailed analysis of the function $f(w)$ shows that it contains the same Cherenkov poles $\left\{\omega_{l}^{\text {Ch }}\right\}$ as the incident field (12) does (see the Appendix C). Therefore, Cherenkov radiation penetrated all vacuum areas of the structure which is described by contribution of these poles (residues) can be easily calculated, similar to Eq. (15). For example, Cherenkov radiation penetrated areas 2 and 3 (which is of most interest) can be expressed as follows:

$$
H_{\phi}^{\mathrm{Ch}(\alpha)}(r, z, t)=\sum_{l=1}^{\infty} H_{\phi l}^{\mathrm{Ch}(\alpha)}(r, z, t)
$$

$$
H_{\phi l}^{\mathrm{Ch}(\alpha)}(r, z, t)=2 \operatorname{Re}\left[-2 \pi i \operatorname{Res}_{\omega_{l}^{\mathrm{Ch}}} H_{\omega \phi}^{(\alpha)} e^{-i \omega_{l}^{\mathrm{Ch}} t}\right],
$$

$\alpha=2,3$ (means corresponding subarea of the structure). Note that each summand in Eq. (40) depends on Fourier transform of the scattered field $H_{\omega \phi}^{(\alpha)}$ which is presented as infinite series over waveguide modes [Eqs. (18) and (19)]. Since we suppose that (40) describes radiation, these series should be truncated to contain only propagating modes for given Cherenkov frequency.

Let us discuss the procedure to calculate the contribution of $\omega_{l}^{\text {Ch }}$ for given $l$. Since $\left\{A_{m}\right\}$ and $\left\{C_{n}\right\}$ have the pole for $\omega=\omega_{l}^{\mathrm{Ch}}$, then

$$
H_{\omega \phi}^{(\alpha)} \approx \frac{\operatorname{Res}_{\omega_{l}^{\mathrm{Ch}}} H_{\omega \phi}^{(\alpha)}}{\omega-\omega_{l}^{\mathrm{Ch}}}, \quad \omega \rightarrow \omega_{l}^{\mathrm{Ch}} .
$$

Here, the term in the numerator is the residue to be found. If we suppose that dielectric possesses some small dissipation, $\varepsilon=\varepsilon^{\prime}+i \varepsilon^{\prime \prime}$, then Cherenkov pole also becomes complex:

$$
\omega_{l}^{\mathrm{Ch}}=\omega_{l}^{\mathrm{Ch} /}+i \omega_{l}^{\mathrm{Ch} / \prime}, \quad \omega_{l}^{\mathrm{Ch} / \prime}<0 .
$$

Using numerical procedure described in Appendix B, we calculate $H_{\omega \phi}^{(\alpha)}$ for $\omega=\omega_{l}^{\mathrm{Ch} /}$, therefore

$$
\operatorname{Res}_{\omega_{l}^{\mathrm{Ch}}} H_{\omega \phi}^{(\alpha)}=-\left.i \omega_{l}^{\mathrm{Ch} \prime \prime} H_{\omega \phi}^{(\alpha)}\right|_{\omega=\omega_{l}^{\mathrm{Ch}}}
$$

In this way one can calculate contributions of all the essential Cherenkov poles relatively simple and fast. Corresponding examples are represented below in Sec. III.

\section{NUMERICAL RESULTS}

Here we present numerical results obtained via rigorous formulas of the previous section and results of direct numerical simulation in CST PS ${ }^{\circledR}$ package (with the use of wakefield solver). For simulation, we constructed the model with finite $L_{v}$ and $L_{d}$ (see Fig. 1) and open boundary conditions for $z=-L_{d}, z=L_{v}$. Also small finite thickness of the inner waveguide wall $d_{w} \ll a, b$ was taken into account in simulations, i.e., we supposed that coaxial area 2 is determined by inequality $b+d_{w}<r<a, z<0$. The adaptive meshing procedure was utilized to obtain optimal simulation parameters and stable results, this point will be explained below using representative example (see Fig. 4).

First, we clarify the statement concerning the frequency spectrum of the fields in vacuum areas of the structure. Figure 2 shows position of the first seven shifted zeros $\Gamma_{m}$ and "unshifted" zeros $\gamma_{z m}^{(1)}$ on the complex plane calculated for three Cherenkov frequencies (14). These results are supplemented by Table I where corresponding numerical values $\left(\gamma_{z m}^{(1)} / \Gamma_{m}\right)$ are presented with the 0.01 relative 


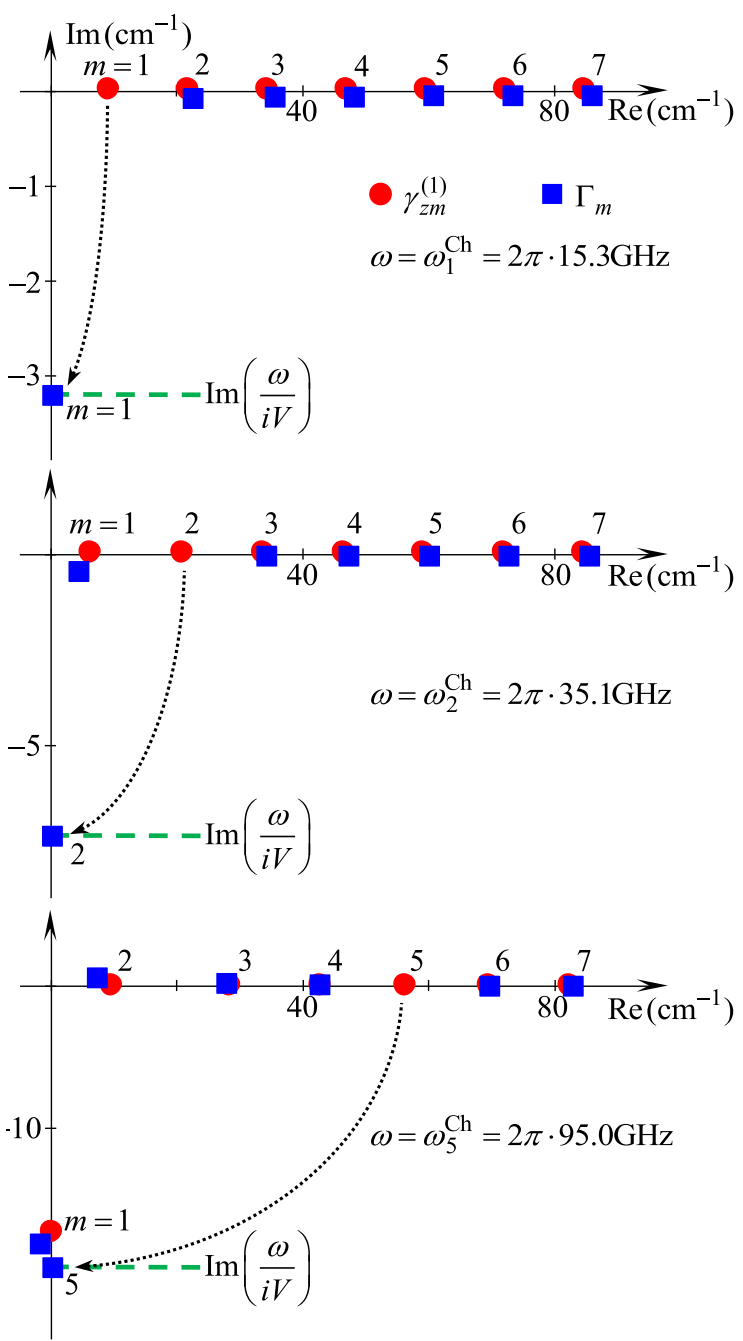

FIG. 2. Comparison between shifted and "unshifted" zeros $\gamma_{z m}^{(1)}\left(\mathrm{cm}^{-1}\right)$ and $\Gamma_{m}\left(\mathrm{~cm}^{-1}\right), m=1,2, \ldots 7$ for three frequencies of Cherenkov radiation $\omega=\omega_{l}^{\mathrm{Ch}}, l=1,2,5$. Structure parameters are: $b=0.25 \mathrm{~cm}, a=0.9 \mathrm{~cm}, \varepsilon=10+i 10^{-5}$.

accuracy. Note that since a small dissipation in dielectric is taken into account $\left(\varepsilon^{\prime \prime} / \varepsilon^{\prime}=10^{-6}\right)$, Cherenkov frequencies are complex values, as Eq. (42) indicates. The discussed calculations are performed for $\omega=\omega_{l}^{\mathrm{Ch} \prime}$.
As one can see from Fig. 2, the majority of presented $\Gamma_{m}$ are weakly shifted with respect to $\gamma_{z m}^{(1)}$ excluding $\Gamma_{l}$ (with the number of Cherenkov frequency under consideration). This $\Gamma_{l}$ is shifted dramatically so that it becomes purely imaginary while initial $\gamma_{z l}^{(1)}$ was purely real. Moreover, one can learn from Table I that the equality

$$
\Gamma_{l}\left(\omega_{l}^{\mathrm{Ch}}\right) \approx \omega_{l}^{\mathrm{Ch}} /(i V)
$$

is fulfilled with high accuracy, because

$$
\frac{\omega_{1}^{\mathrm{Ch}}}{i V} \approx-3.21 i, \quad \frac{\omega_{2}^{\mathrm{Ch}}}{i V} \approx-7.36 i, \quad \frac{\omega_{5}^{\mathrm{Ch}}}{i V} \approx-19.91 i .
$$

Note that in Eq. (45) we present numerical values with 0.01 relative accuracy, similar to Table I.

As it is shown in Appendix B, Eq. (45) leads to conclusion that all sets of unknown coefficients possess poles for Cherenkov frequencies. Contribution of these poles in vacuum areas of the structure can be calculated using Eq. (43).

\section{A. Single bunch field}

Here we present numerical results illustrating the field behavior in different subareas of the structure. For all figures, radius of the inner waveguide is the same, $b=0.25 \mathrm{~cm}$. The structure is excited by single relativistic Gaussian bunch (5).

For Figs. 3-8, the bunch length $\sigma$ is chosen so that only the first Cherenkov frequency $\omega_{1}^{\mathrm{Ch}}$ lies within essential part of frequency spectrum $\left[0, \omega_{\max }\right]$ (7) while higher Cherenkov frequencies are suppressed by attenuating Gaussian term (6). In this case we expect monochromatic both Cherenkov radiation in the inner waveguide and Cherenkov radiation penetrating areas 2 and 3 .

Figure 3 shows transverse electric field $E_{r}$ from the probe located in the inner waveguide for the case of relatively large permittivity, $\varepsilon=10$, and $a=0.5 \mathrm{~cm}$. The part of the signal enclosed in the dashed line rectangle $(0.4 \mathrm{~ns}<t<1.1 \mathrm{~ns})$ should be interpreted as the field of Cherenkov radiation. The Fourier spectrum of this part of the signal shown in the inset of Fig. 3 has a strong peak

TABLE I. Comparison between shifted and "unshifted" zeros $\left(\gamma_{z m}^{(1)} / \Gamma_{m}\right)$ for $m=1,2, \ldots 7$ and three Cherenkov frequencies $\omega_{l}^{\mathrm{Ch}}, l=1,2,5$.

\begin{tabular}{lccc}
\hline \hline$m$ & $\omega_{1}^{\mathrm{Ch}}$ & $\omega_{2}^{\mathrm{Ch}}$ & $\omega_{5}^{\mathrm{Ch}}$ \\
\hline 1 & $9.07 /-3.21 i$ & $6.19 / 4.31-0.45 i$ & $-17.43 i /-2.01-18.18 i$ \\
2 & $21.85 / 22.53-0.08 i$ & $20.82 /-7.36 i$ & $9.55 / 7.11+0.54 i$ \\
3 & $34.47 / 35.38-0.08 i$ & $33.82 / 34.17-0.05 i$ & $28.32 / 27.76+0.16 i$ \\
4 & $47.06 / 48.10-0.07 i$ & $46.59 / 47.13-0.06 i$ & $42.76 / 42.55+0.06 i$ \\
5 & $59.64 / 60.77-0.06 i$ & $59.27 / 59.95-0.06 i$ & $56.31 /-19.91 i$ \\
6 & $72.21 / 73.41-0.05 i$ & $71.91 / 72.69-0.05 i$ & $69.49 / 69.63-0.04 i$ \\
7 & $84.79 / 86.03-0.05 i$ & $84.53 / 85.38-0.05 i$ & $82.48 / 82.73-0.06 i$ \\
\hline \hline
\end{tabular}




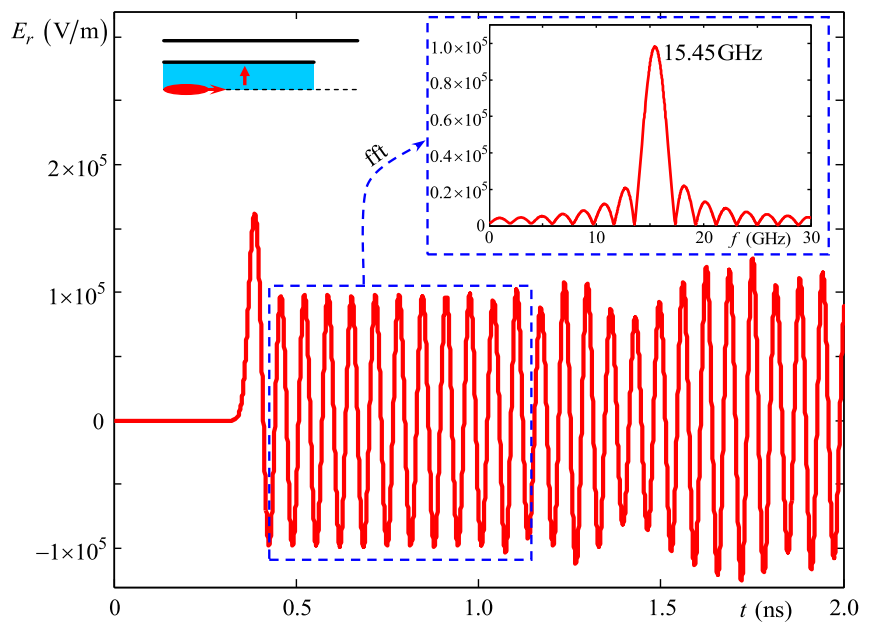

FIG. 3. Behavior of the electric field $E_{r}$ over time (CST result) on the probe in the inner waveguide: $z=-1 \mathrm{~cm}, r=0.125 \mathrm{~cm}$. Time $t=0$ corresponds to the moment when bunch center is in the plane $z=0$. Structure parameters: $b=0.25 \mathrm{~cm}, a=0.5 \mathrm{~cm}$, $\varepsilon=10, L_{d}=35 \mathrm{~cm}, L_{v}=50 \mathrm{~cm}$, inner waveguide wall thickness is $0.001 \mathrm{~cm}$. Gaussian bunch parameters: $q=1 \mathrm{nC}$, $\beta=0.9999, \sigma=0.5 \mathrm{~cm}$.

for frequency $15.45 \mathrm{GHz}$, this is Cherenkov radiation frequency obtained in the numerical experiment. For shortness, this result for simulated frequency will be referred to as "experimental" result throughout this section.

It should be noted that mentioned peak is used for adaptive meshing procedure in CST simulation: mesh is refined (number of lines per wavelength is increased) until the position of the peak becomes stable, i.e., relative difference in position is less than 0.002 for two consequent passes. Figure 4 illustrates this procedure. It shows typical dependence of the experimental Cherenkov frequency on the number of mesh lines per wavelength (this is standard parameter defining mesh density in CST; wavelength is

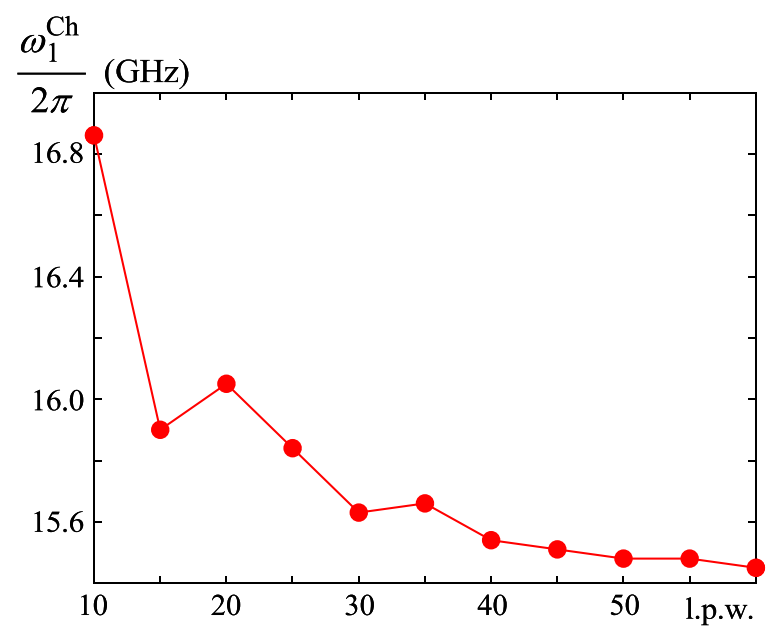

FIG. 4. Dependence of the simulated first Cherenkov frequency $\omega_{1}^{\mathrm{Ch}} /(2 \pi)(\mathrm{GHz})$ on number of lines per wavelength (1.p.w.). Problem parameters are the same as in Fig. 3.
TABLE II. Comparison between analytical and experimental (CST) first Cherenkov frequency $\omega_{1}^{\text {Ch }}$ (Analytical/Experimental) for $b=0.25 \mathrm{~cm}$.

\begin{tabular}{ccc}
\hline \hline & $\varepsilon=10$ & $\varepsilon=2$ \\
\hline$a=0.5 \mathrm{~cm}$ & $15.31 \mathrm{GHz} / 15.45 \mathrm{GHz}$ & $45.9 \mathrm{GHz} / 46.13 \mathrm{GHz}$ \\
$a=0.9 \mathrm{~cm}$ & $15.31 \mathrm{GHz} / 15.48 \mathrm{GHz}$ & $45.9 \mathrm{GHz} / 46.27 \mathrm{GHz}$ \\
\hline \hline
\end{tabular}

understood as the minimal wavelength which corresponds to $\left.\omega_{\max }\right)$. As one can see, for rare mesh the experimental frequency is considerably larger compared to the theoretical value $(15.31 \mathrm{GHz})$. For 60 lines per wavelength the relative difference is sufficiently small therefore procedure is stopped, the obtained frequency differs from the theoretical by less then one percent. Comparison between first Cherenkov frequencies, theoretical, and experimental, for all structures discussed below is shown in Table II. Theoretical value of Cherenkov frequency does not depend on radius of the outer waveguide $a$, but this is not the case for simulations due to the change in mesh with change in $a$. In all considered cases, relative difference between theoretical and experimental values is around 1 percent. As one can see below, this small difference matters in comparison of the field behavior.

Figure 5 shows CST simulated signal from the probe located in area 2 of the structure with $\varepsilon=10$ and $a=0.5 \mathrm{~cm}$. Solid (green) line corresponds to the field obtained via simulation in CST PS ${ }^{\circledR}$ code. According to the CST curve, with an increase in time $t$, a strong peak corresponding to the "image" of the bunch can be seen first (this effect was discussed in details in the case of similar vacuum structure in Ref. [25]). After that, some transition process connected with diffraction radiation occurs. For large enough time $(t \gtrsim 0.5 \mathrm{~ns})$ we see the stationary harmonic process. Top inset in Fig. 5 shows magnified part of the CST curve compared with theoretical curve

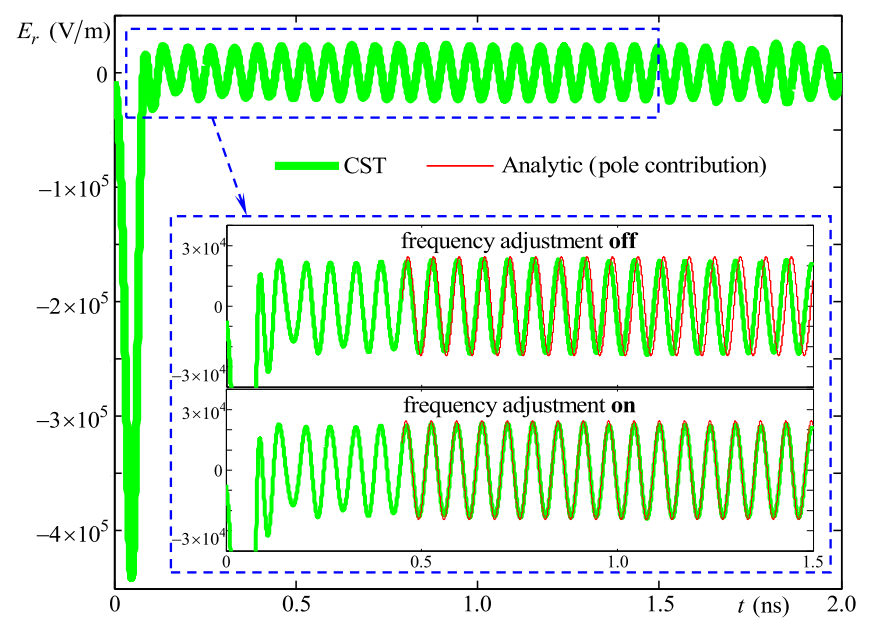

FIG. 5. Behavior of the electric field $\left(E_{r}\right)$ over time on the probe in the coaxial area: $z=-1 \mathrm{~cm}, r=0.35 \mathrm{~cm}$. Structure and bunch parameters are the same as in Fig. 3. 


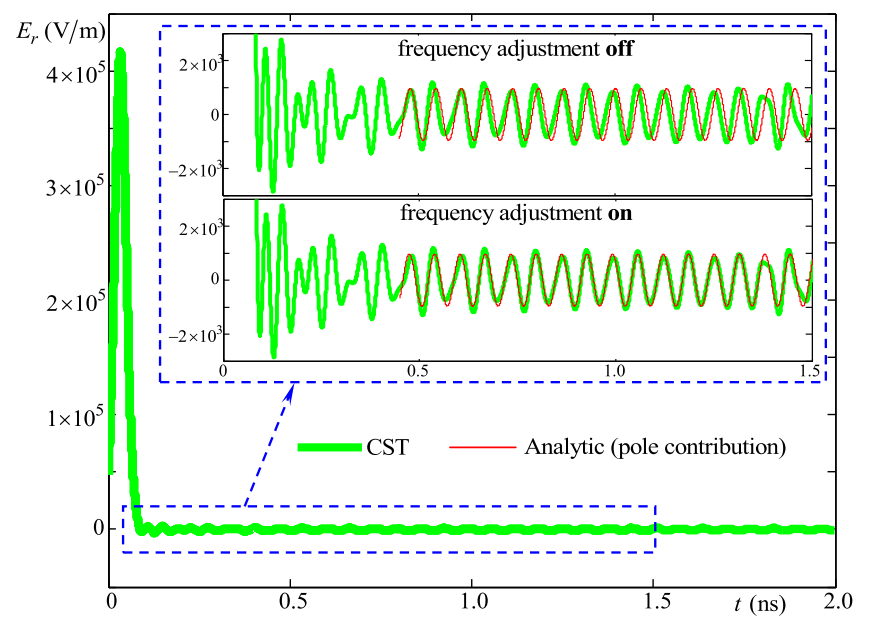

FIG. 6. Behavior of the electric field $\left(E_{r}\right)$ over time on the probe in the wide vacuum waveguide: $z=1 \mathrm{~cm}, r=0.35 \mathrm{~cm}$. Structure and bunch parameters are the same as in Fig. 3.

corresponding to contribution of the first Cherenkov pole, i.e., summand with $l=1$ and $\alpha=2$ in (39) (red line). Magnitudes correlate well but due to the difference in frequency the curves diverge for large enough time. If we manually adjust the frequency in analytical formulas, i.e., substitute the analytical Cherenkov frequency with the experimental one (see Table II), we will obtain an excellent coincidence between the curves shown in the bottom inset in Fig. 5. The described frequency substitution is further called "frequency adjustment." Based on presented comparison and the tendency for experimental Cherenkov frequency (see Fig. 4) one can conclude on both correctness of the used analytical approach and stable operation of the simulation code for fine enough mesh.

Figure 6 shows similar comparison (for the same structure) but for probe located in the area 3 (wide vacuum waveguide). Again, after the frequency adjustment applied the curves correlate very well. Further for all figures the frequency adjustment will be used by default. Note that for given $a$ even the first mode in area 3 is evanescent therefore magnitude of Cherenkov radiation is extremely small. Figure 7 illustrates the case of $\varepsilon=10$ and larger radius of the outer waveguide, $a=0.9 \mathrm{~cm}$. In this case, Cherenkov radiation penetrated area 3 is more expressed and again it is described very well by analytical formulas.

Figure 8, shows signals from symmetrical probes in coaxial and vacuum waveguide areas for the structure with lower permittivity $(\varepsilon=2)$ and correspondingly higher Cherenkov frequency. Again, one can see that pole contribution calculated theoretically describes Cherenkov radiation penetrated vacuum parts of the structure very well.

\section{B. Bunch train field}

Here we illustrate the possibilities of the described approach for calculation of Cherenkov radiation at highorder modes. According to the idea of beam-driven $\mathrm{THz}$

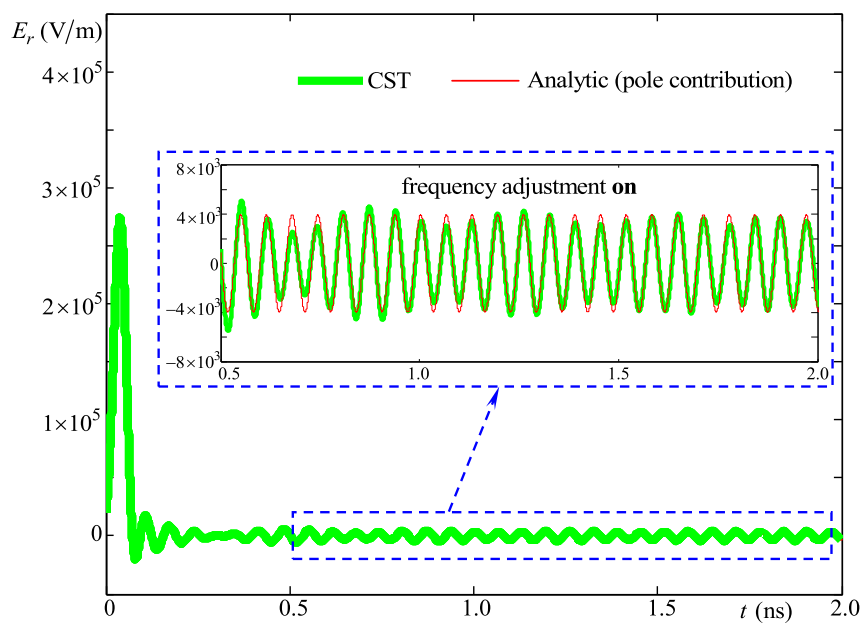

FIG. 7. Behavior of the electric field $\left(E_{r}\right)$ over time on the probe in the wide vacuum waveguide: $z=1 \mathrm{~cm}, r=0.35 \mathrm{~cm}$. Outer waveguide radius $a=0.9 \mathrm{~cm}$, other parameters are the same as in Fig. 3.

source described in Sec. I, THz frequencies can be generated in $\mathrm{mm}$ - or sub-mm-sized waveguides by charged particle bunches with proper charge modulation, i.e. by bunch trains [7]. If we denote by $\tilde{\eta}_{G}$ the Fourier spectrum of a single Gaussian bunch $\eta_{\mathrm{G}}$ (5), we obtain from (4):

$$
\tilde{\eta}_{\mathrm{G}}(\xi)=(2 \pi)^{-1} \exp \left(-\xi^{2} \sigma^{2} / 2\right)
$$

The sequence of $2 M+1$ identical Gaussian bunches spaced by $L$ and carrying the same total charge has the following longitudinal charge distribution:

$$
\eta_{\mathrm{Seq}}(z-V t)=\frac{1}{2 M+1} \sum_{m=-M}^{M} \eta_{\mathrm{G}}(z-V t+m L),
$$

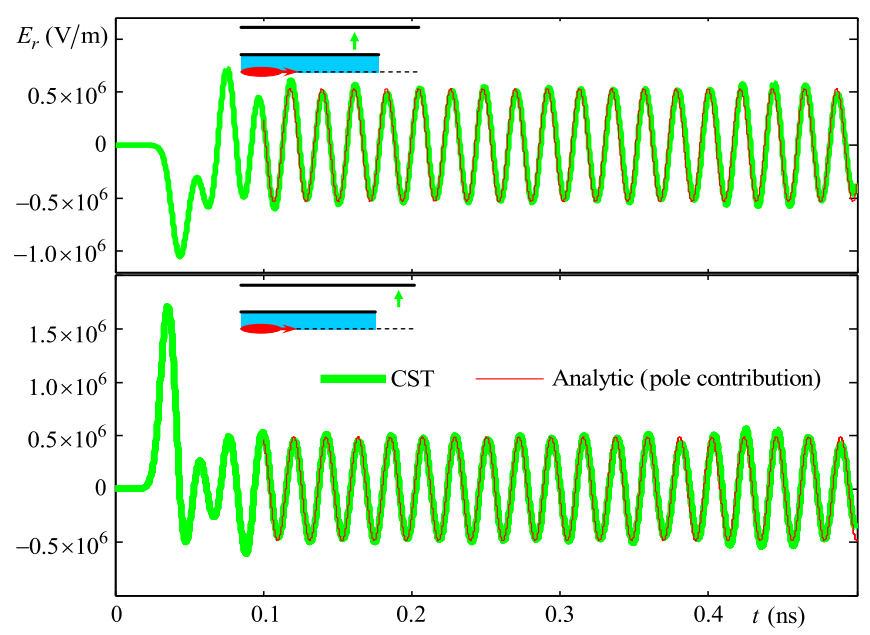

FIG. 8. Behavior of the electric field $E_{r}$ over time on the symmetrical probes in areas 2 and $3: z= \pm 1 \mathrm{~cm}, r=0.35 \mathrm{~cm}$. Permittivity $\varepsilon=2$, other parameters are the same as in Fig. 3. 


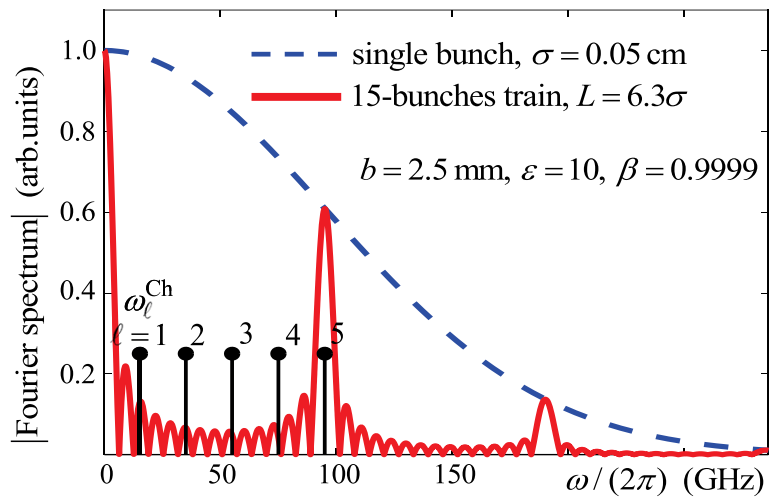

FIG. 9. Fourier spectrum of a single Gaussian bunch and that of a 15-bunches train with $L>2 \sigma$ spacing. Black markers show Cherenkov frequencies $\omega_{l}^{\mathrm{Ch}}$. Bunches parameters: $q=1 \mathrm{nC}$, $\beta=0.9999, \sigma=0.05 \mathrm{~cm}, L=6.3 \sigma$. Cherenkov frequencies are calculated for the inner waveguide with $b=0.25 \mathrm{~cm}$ filled with dielectric with $\varepsilon=10$.

and the following spectrum, in accordance with (4):

$$
\begin{aligned}
\tilde{\eta}_{\mathrm{Seq}}(\xi)= & \frac{\tilde{\eta}_{\mathrm{G}}(\xi)}{2 M+1} \\
& \times\left[1+2 \cos \left(\frac{\xi L(M+1)}{2}\right) \frac{\sin (\xi L M / 2)}{\sin (\xi L / 2)}\right] .
\end{aligned}
$$

Figure 9 shows comparison of a single Gaussian bunch Fourier spectrum (46) with the spectrum of a bunch train (48) of $15(M=7)$ identical bunches with spacing $L>2 \sigma$. Both functions are calculated for $\xi=\omega / V$. Cherenkov frequencies (14) for a mm-sized waveguide are also shown. Parameters $\sigma$ and $L$ are chosen so that the bunch train spectrum has the expressed maximum exactly at Cherenkov frequency $\omega_{5}^{\mathrm{Ch}}$. Therefore, this bunch train excites effectively the 5th Cherenkov mode with the frequency around $0.1 \mathrm{THz}$ falling in the sub-THz range. In the same manner, other Cherenkov frequencies can be generated separately.

It should be noted that simulation of EM field produced by such bunch trains is rather complicated in CST PS package. In particular, according to Fig. 4, number of mesh lines per wavelength required for adequate convergence should be increased considerably. Another issue here is the need for manual determination of bunch profile. These issues will be discussed below in more details. On the contrary, the presented analytical technique allows computation of the EM field properties relatively simple and fast which is illustrated below by Figs. 10 and 11 .

Figures 10 and 11 show behavior of $E_{r}$ component of Cherenkov radiation at high-order Cherenkov frequency in vacuum regions of the structure. Recall that this radiation is generated in the inner waveguide and penetrated vacuum sections of the structure by means of diffraction mechanism. Figure 10 illustrates the case of mm-sized structure resulting in sub-THz Cherenkov radiation at 5th Cherenkov frequency, $\omega_{5}^{\mathrm{Ch}} \approx 2 \pi \cdot 95 \mathrm{GHz}$. Figure 10(a) shows $E_{r}$ field in three cross sections of the coaxial region (area 2 in Fig. 1) while Fig. 10(b) shows $E_{r}$ field in three cross

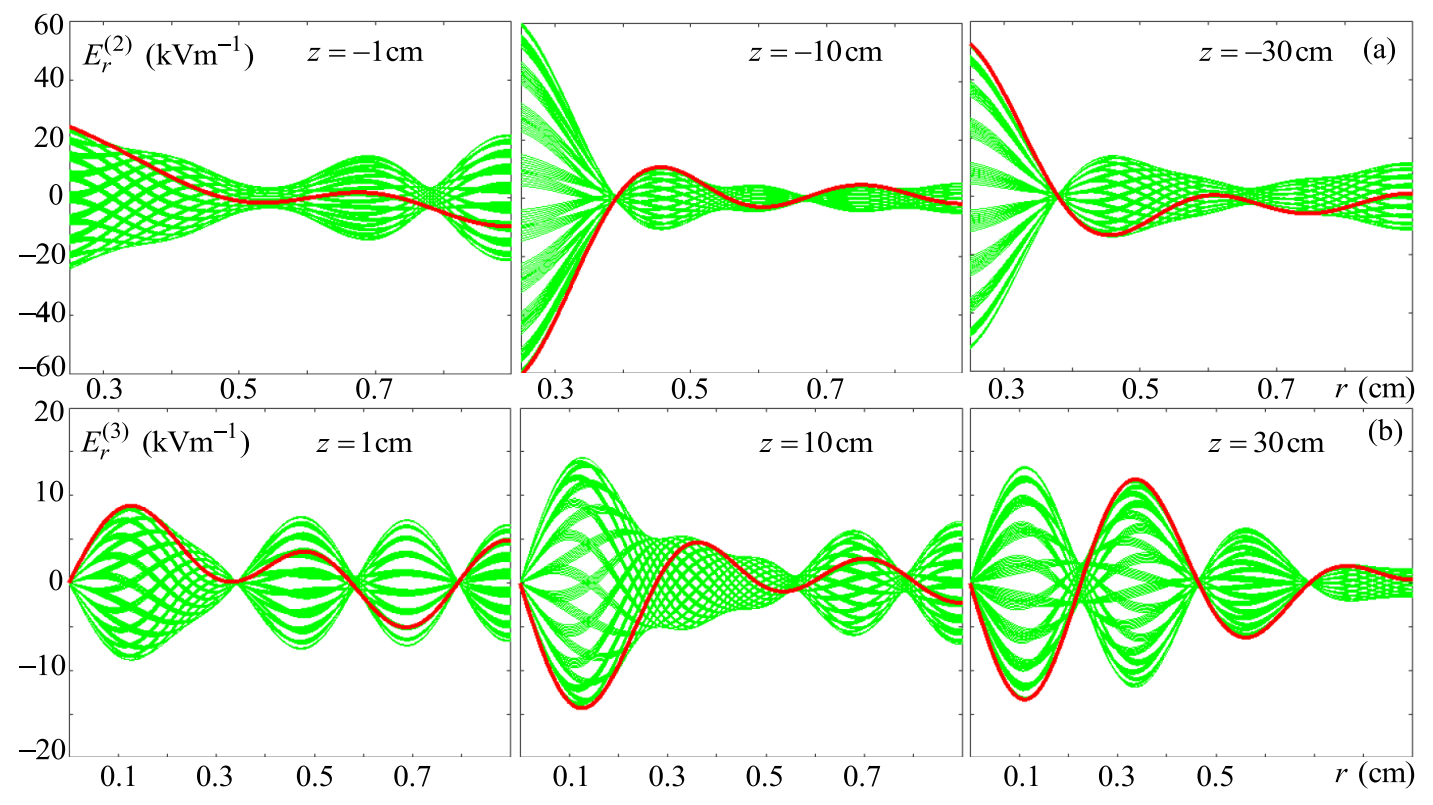

FIG. 10. Cherenkov radiation field $\left(E_{r}\right.$ component) at 5 th Cherenkov frequency, $\omega_{5}^{\mathrm{Ch}} \approx 2 \pi \cdot 95 \mathrm{GHz}$, in vacuum regions of the structure: in the coaxial area (a) and in wide vacuum waveguide (b). Each thin (green) curve shows the $E_{r}^{\text {Ch }}$ as a function of $r$ at given time moment $t$ and given $z$. In total, each plot contains 151 curves covering the $1.5 \mathrm{~ns}$ time range with $0.01 \mathrm{~ns}$ interval. Solid (red) curve corresponds to the global field maximum over the cross section. Parameters of the structure and the bunch train are the same as in Fig. 9 so that the 5 th Cherenkov frequency is effectively generated. 

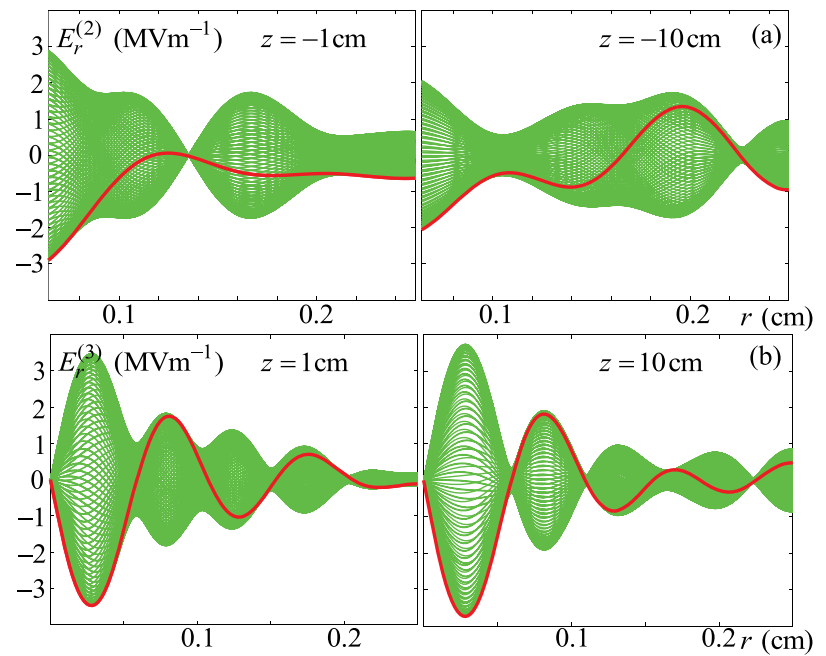

FIG. 11. Cherenkov radiation field ( $E_{r}$ component) at the 3-rd Cherenkov frequency, $\omega_{3}^{\mathrm{Ch}} \approx 2 \pi \cdot 386 \mathrm{GHz}$, in vacuum regions of the structure: in the coaxial area (a) and in wide vacuum waveguide (b). Curves notations are the same as in Fig. 10. Parameters of the structure: $b=0.064 \mathrm{~cm}, a=0.25 \mathrm{~cm}, \varepsilon=3.8$ ( $b$ and $\varepsilon$ correspond to ones used in [5]). Bunch train parameters $q=1 \mathrm{nC}, \beta=0.9999, \sigma=0.012 \mathrm{~cm}, L=6.45 \sigma$ are chosen so that the 3rd Cherenkov frequency is effectively generated.

sections of the wide vacuum waveguide (area 3 in Fig. 1). Each thin (green) curve shows the $E_{r}^{\mathrm{Ch}}$ as a function of $r$ at a given time moment $t$ and given $z$. In total, each plot contains 151 curves covering the $1.5 \mathrm{~ns}$ time range with $0.01 \mathrm{~ns}$ interval. The highlighted solid (red) curve is the curve which provides the maximum field magnitude over the cross section. Since at the given Cherenkov frequency both coaxial waveguide and wide vacuum waveguide supports 5 propagating modes, field behavior is rather complicated. As one can see, maximum field in coaxial region is always on the inner waveguide wall. In the wide waveguide, global field maximum is typically at the first or second local maximum.

Figure 11 illustrates the case of sub-mm inner waveguide (its size and dielectric filling corresponds to one of the structures used in [5]) resulting in Cherenkov radiation in the lower $\mathrm{THz}$ range (about $0.39 \mathrm{THz}$ ). Compared to the Fig. 10, field magnitude is about 1 order larger in this case while aforementioned properties of field distribution are mainly the same.

It should be underlined that simulation time consumed by CST Particle Studio strongly depends on mesh density. As Fig. 4 illustrates, the reasonable agreement in frequency of Cherenkov radiation (for the first Cherenkov frequency around $15 \mathrm{GHz}$ ) is achieved for approximately 60 lines per wavelength. For this mesh density, calculation of the model (with parameters corresponding to those used in Figs. 3, 5, 6, 7 , and 8) typically takes about 20 hours at PC with Intel ${ }^{\circledR}$ Core i7 processor and $32 \mathrm{~Gb}$ memory (with memory usage about $100 \%$ ). On the other hand, our MATLAB code based on analytical formulas typically takes around 1 minute to obtain the curves presented in these figures. Consequently, the analytical approach is approximately $20 \times 60$ times faster.

Moreover, for the case of a bunch train generating a highorder Cherenkov frequency (around $100 \mathrm{GHz}$ for the case shown in Fig. 10 and around $0.4 \mathrm{THz}$ for the case shown in Fig. 11) there is a number of problems with CST simulations. First, there is no standard option to launch a bunch train into the structure. Second, the amount of memory required for realization of appropriate mesh is far beyond typical $32 \mathrm{~Gb}$ of personal computers: 6 times decrease in minimal wavelength results in $6^{3}$ times increase in number of mesh cells. Therefore, the presented analytical approach can be considered as preferable method for analysis of Cherenkov radiation field across the structure.

\section{CONCLUSION AND DISCUSSION}

First, we have considered radiation produced by single Gaussian bunch exiting the open end of a cylindrical waveguide with uniform dielectric filling in the case where this waveguide is put into concentric vacuum infinite waveguide of larger radius ("embedded" structure). Based on residue-calculus technique, we have constructed the rigorous theory of the electromagnetic process in this structure. Based on this theory, Cherenkov radiation exiting from dielectric waveguide into vacuum parts of the structure, which is of essential interest in the context of beam driven radiation sources, can be calculated easily and fast. We also have performed numerical simulation of the process in CST PS code for long enough bunch generating the first Cherenkov frequency only. It has been shown that simulated Cherenkov radiation spectral peak has correct frequency for only dense enough mesh. In this case, numerical and analytical results for Cherenkov radiation coincided very well therefore proving both the correctness of rigorous approach and good convergence of numerical procedure. However, the code based on analytical formulas shows about 1000 times faster performance.

Second, using the aforementioned analytical approach, we have considered generation of high-order Cherenkov modes by modulated bunches (bunch trains) in vacuum regions of the structure. Since trains of short bunches generate relatively high frequencies, correct numerical simulations in CST PS becomes extremely difficult. In this case, the presented rigorous approach allowing convenient and fast analysis of the EM field across the structure will be the preferred (and probably the only reliable) method of investigation.

Typically, the structure should have a vacuum channel for passage of the bunch. In the present paper, we have considered the case without channel mainly for the sake of results simplicity. Moreover, as it is known, if channel radius is relatively small (in particular, it should be much smaller then wavelength, see [53] for details), Cherenkov radiation outside the channel is practically the same as in the case without channel. However, for $\mathrm{THz}$ radiation this requirement is difficult to satisfy and the problem with 
finite vacuum channel taken into account should be considered. The presented approach can be generalized for this case without principal difficulties (though the formulas will become more cumbersome).

For possible experiments, for the inner waveguide of the "embedded" structure (Fig. 1) one can use dielectric structures similar to those used in Refs. [5,9,10]. For example, in Ref. [5] these were $\mathrm{SiO}_{2}$ capillaries $(\varepsilon=3.8)$ coated with metal and having outer diameter 400 or 640 microns and lengths up to $15 \mathrm{~cm}$. The inner diameter was 300 or 450 microns, therefore requiring analytical consideration for the case with channel, as discussed above. The bunch with around $3 \mathrm{pC}$ charge was focused to approximately $30 \times 30$ microns in transverse directions allowing passage through the mentioned structures without significant beam losses.

The outer waveguide can represent the walls of the beamline or experimental vacuum chamber, or other more sophisticated solutions mentioned in Sec. I can be realized. One important advantage here is that one can detect the diffracted Cherenkov radiation in the coaxial part of the structure located downstream the particle bunch.

\section{ACKNOWLEDGMENTS}

Authors acknowledge the support from Russian Science Foundation (Grant No. 18-72-10137) at the stage of the paper modification in accordance with reviewers' criticism.

\section{APPENDIX A: INFINITE SYSTEMS FOR MODE DECOMPOSITION COEFFICIENTS}

$$
\left\{\boldsymbol{A}_{\boldsymbol{m}}\right\},\left\{\boldsymbol{B}_{\boldsymbol{m}}\right\}, \text { AND }\left\{\boldsymbol{C}_{\boldsymbol{n}}\right\}
$$

Boundary conditions in the plane $z=0$ result in the following relations:

$$
\begin{array}{ll}
H_{\omega \phi}^{(1)}(r, 0)=H_{\omega \phi}^{(3)}(r, 0) & \text { for } 0 \leq r \leq b, \\
H_{\omega \phi}^{(2)}(r, 0)=H_{\omega \phi}^{(3)}(r, 0) & \text { for } b \leq r \leq a,
\end{array}
$$

$$
\begin{array}{ll}
\frac{\partial H_{\omega \phi}^{(1)}(r, z)}{\varepsilon \partial z}=\left.\frac{\partial H_{\omega \phi}^{(3)}(r, z)}{\partial z}\right|_{z=0} \quad \text { for } 0 \leq r \leq b, \\
\frac{\partial H_{\omega \phi}^{(2)}(r, z)}{\partial z}=\left.\frac{\partial H_{\omega \phi}^{(3)}(r, z)}{\partial z}\right|_{z=0} \quad \text { for } b \leq r \leq a .
\end{array}
$$

To eliminate dependence on $r$, we substitute (17) and (18) into (A1) and (A3), integrate obtained relations over $0<r<b$ with the weight function $r J_{1}\left(r j_{0 p} / b\right), p=$ $1,2, \ldots$ and utilize the following properties [57]:

$$
\int_{0}^{b} r J_{1}\left(\frac{r j_{0 p}}{b}\right) J_{1}\left(\frac{r j_{0 m}}{b}\right) d r=\frac{b^{2} J_{1}^{2}\left(j_{0 p}\right) \delta_{p m}}{2},
$$

(here $\delta_{p m}$ is the Kronecker symbol, $m=1,2, \ldots$ ),

$$
\int_{0}^{b} r J_{1}\left(\frac{r j_{0 p}}{b}\right) H_{1}^{(0)}(r \tilde{s}) d r=\frac{\frac{2 i j_{0 p}}{\pi b \tilde{s}}-b \tilde{s} H_{0}^{(1)}(b \tilde{s}) J_{1}\left(j_{0 p}\right)}{\tilde{s}^{2}-\left(j_{0 p} / b\right)^{2}},
$$

$$
\int_{0}^{b} r J_{1}\left(\frac{r j_{0 p}}{b}\right) J_{1}\left(\frac{r j_{0 m}}{a}\right) d r=\frac{b \frac{j_{0 m}}{a} J_{0}\left(\frac{b j_{0 m}}{a}\right) J_{1}\left(j_{0 p}\right)}{\left(j_{0 p} / b\right)^{2}-\left(j_{0 m} / a\right)^{2}},
$$

$\tilde{s}=s$ or $\tilde{s}=s_{0}$. Taking into account that

$$
\begin{gathered}
\left(j_{0 p} / b\right)^{2}-\left(j_{0 m} / a\right)^{2}=\left(\gamma_{z p}^{(1)}\right)^{2}-\left(\gamma_{z m}^{(3)}\right)^{2} \\
s_{0}^{2}-\left(j_{0 p} / b\right)^{2}=(i \omega / V)^{2}-\left(\gamma_{z p}^{(1)}\right)^{2},
\end{gathered}
$$

one can obtain after algebraic manipulations:

$$
\begin{gathered}
\sum_{m=1}^{\infty}\left[\frac{\tilde{A}_{m}}{\gamma_{z m}^{(3)}-\gamma_{z p}^{(1)}}+\frac{\tilde{A}_{m} R_{p}}{\gamma_{z m}^{(3)}+\gamma_{z p}^{(1)}}\right]+\frac{\frac{i q}{2 c b}}{J_{1}\left(j_{0 p}\right)}\left[\left(\frac{\pi b^{2} s_{0}^{2} h_{0} J_{1}\left(j_{0 p}\right)}{2 j_{0 p}}-1\right)\left(F_{v p}^{-}+R_{p} F_{v p}^{+}\right)+F_{d p}^{+}+R_{p} F_{d p}^{-}\right]=0, \\
\sum_{m=1}^{\infty}\left[\frac{\tilde{A}_{m}}{\gamma_{z m}^{(3)}+\gamma_{z p}^{(1)}}+\frac{\tilde{A}_{m} R_{p}}{\gamma_{z m}^{(3)}-\gamma_{z p}^{(1)}}\right]+\frac{\frac{i q}{2 c b}}{J_{1}\left(j_{0 p}\right)}\left[\left(\frac{\pi b^{2} s_{0}^{2} h_{0} J_{1}\left(j_{0 p}\right)}{2 j_{0 p}}-1\right)\left(R_{p} F_{v p}^{-}+F_{v p}^{+}\right)+R_{p} F_{d p}^{+}+F_{d p}^{-}\right]=\frac{4 \gamma_{z p}^{(1)} \kappa_{z p}^{(1)} \tilde{B}_{p}}{\kappa_{z p}^{(1)}+\varepsilon \gamma_{z p}^{(1)}},
\end{gathered}
$$

where $h_{0}$ is given by Eq. (37),

$$
\begin{gathered}
\tilde{A}_{m}=A_{m} j_{0 m} J_{0}\left(b j_{0 m} / a\right) / a, \\
\tilde{B}_{p}=B_{p} b J_{1}\left(j_{0 p}\right) / 2,
\end{gathered}
$$

$R_{p}, F_{d p}^{ \pm}$and $F_{v p}^{ \pm}$are given by Eqs. (30), (31), and (32), correspondingly.
In a similar way, we substitute (18) and (19) into (A2) and (A4), integrate these relations over the interval $b<$ $r<a$ with the weight function $r Z_{p}\left(r \chi_{p}\right)$ and utilize the property

$$
\int_{b}^{a} r Z_{m}\left(r \chi_{m}\right) Z_{p}\left(r \chi_{p}\right) d r=\delta_{p m} I_{p}
$$




$$
I_{p}=\frac{a^{2}}{2} Z_{p}^{2}\left(a \chi_{p}\right)-\frac{b^{2}}{2} Z_{p}^{2}\left(b \chi_{p}\right)
$$

and formulas analogous to (A6) and (A7). Taking into account that

$$
\begin{gathered}
\left(j_{0 m} / a\right)^{2}-\chi_{p}^{2}=\left(\gamma_{z m}^{(3)}\right)^{2}-\left(\gamma_{z p}^{(2)}\right)^{2}, \\
s_{0}^{2}-\chi_{p}^{2}=(i \omega / V)^{2}-\left(\gamma_{z p}^{(2)}\right)^{2},
\end{gathered}
$$

after a series of algebraic manipulations one obtains

$$
\begin{gathered}
\sum_{m=1}^{\infty} \frac{\tilde{A}_{m}}{\gamma_{z m}^{(3)}-\gamma_{z n}^{(2)}}+\frac{i q}{2 c} \frac{i s_{0}^{2} h_{0}}{\frac{\omega}{i V}-\gamma_{z n}^{(2)}}=0, \\
\sum_{m=1}^{\infty} \frac{\tilde{A}_{m}}{\gamma_{z m}^{(3)}+\gamma_{z n}^{(2)}}+\frac{i q}{2 c} \frac{i s_{0}^{2} h_{0}}{\frac{\omega}{i V}+\gamma_{z n}^{(2)}}=-2 \gamma_{z p}^{(2)} \tilde{C}_{n},
\end{gathered}
$$

where $n=0,1, \ldots$,

$\tilde{C}_{0}=C_{0} \ln (a / b), \quad \tilde{C}_{p}=C_{p} I_{p}\left[b Z_{p}\left(b \chi_{p}\right)\right]^{-1}$.

Note that the case $n=0$ is obtained by integration of (A2) and (A4) over $b<r<a$ without any weight function.

In the issue, we obtain four infinite systems (A10), (A11), (A18), and (A19). These systems can be solved simultaneously using the residue-calculus technique [12,23-25]. This procedure is described in Appendix B.

\section{APPENDIX B: CONSTRUCTING THE FUNCTION $f(w)$ AND SOLVING INFINITE SYSTEMS}

In accordance with the residue-calculus technique, to solve systems (A10), (A11), (A18), and (A19), let us consider the following Cauchy-type integrals over the infinite radius circle $C_{\infty}$ :

$\oint_{C_{\infty}}\left[\frac{f(w)}{w \mp \gamma_{z p}^{(1)}}+\frac{R_{p} f(w)}{w \pm \gamma_{z p}^{(1)}}\right] d w=\oint_{C_{\infty} w \mp \gamma_{z n}^{(2)}} \frac{f(w) d w}{w}$

where $f(w)$ is a complex-valued function that should be found. These integrals equal zero because we suppose that $f(w)$ vanishes for $|w| \rightarrow \infty$. Next step is constructing $f(w)$ so that it has certain specific zeros, poles, and behavior for $|w| \rightarrow \infty$. To solve this problem, it is useful to have in mind

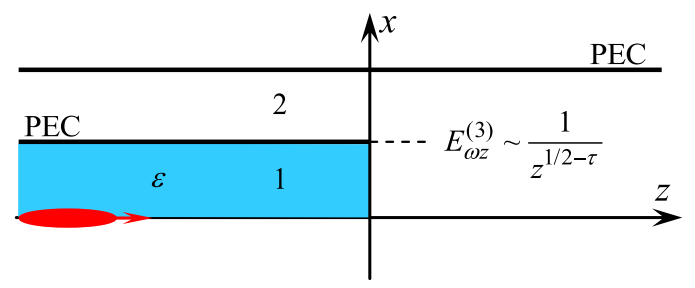

FIG. 12. Correct physical behavior of the longitudinal electric field near the sharp edge $r=b, z \rightarrow+0$ (Meixner edge condition), $\tau=\pi^{-1} \operatorname{asin} \frac{\varepsilon-1}{2 \varepsilon+2}$.

the infinite systems and their solution for the corresponding vacuum problem (with the same geometry and permittivity $\varepsilon=1$ [25]) and point out the differences. First, in the case under consideration, systems (A10) and (A11) are more complicated while systems (A18) and (A19) are the same. Second, the singularity of the longitudinal electric field near the sharp edge $r=b, z \rightarrow+0$,

$$
E_{\omega z}^{(3)} \sim 1 / z^{1 / 2-\tau}, \quad \sin \pi \tau=(\varepsilon-1) /(2 \varepsilon+2),
$$

becomes weaker in the presence of dielectric [12,23] (see Fig. 12) compared to the vacuum case where we have $E_{\omega z}^{(3)} \sim z^{-1 / 2}$ near this edge.

Taking into account these points, one should construct $f(w)$ so that: (i) $f(w)$ is regular in complex plane $w$ excluding first-order poles $w=\gamma_{z p}^{(3)}$ and a pole $w=-i \omega / V$; (ii) $f(w)$ has first-order zeros $w=\gamma_{z n}^{(2)}$ and $w=\Gamma_{m}$, $\Gamma_{m} \neq \gamma_{z m}^{(1)}$; (iii) the residue $\operatorname{Res}_{-i \omega / V} f(w)=-q s_{0}^{2} h_{0} /(2 c)$; (iv) $f(w) \underset{|w| \rightarrow \infty}{\rightarrow} w^{-(\tau+1 / 2)}$, where $\sin (\pi \tau)=\frac{\varepsilon-1}{2 \varepsilon+2}$. Aforementioned differences from vacuum case are taken into account by items (ii) and (iv). In the issue, one can write:

$f(w)=P \frac{\left(w-\gamma_{z 0}^{(2)}\right) \prod_{s=1}^{\infty}\left(1-\frac{w}{\gamma_{z s}^{(2)}}\right) \prod_{p=1}^{\infty}\left(1-\frac{w}{\Gamma_{p}}\right)}{\left(w-\frac{\omega}{i V}\right) \prod_{m=1}^{\infty}\left(1-\frac{w}{\gamma_{z m}^{(3)}}\right)} Q(w)$.

Here $P$ is unknown constant and $Q(w)$ is some regular function providing algebraic (instead of exponential) behavior of $f(w)$ for $|w| \rightarrow \infty$. It can be chosen in the same way as in the vacuum case, in accordance with Eq. (35).

Considering integrals (B1) and calculating them using (B3) and the residue theorem, we obtain:

$$
\sum_{m=1}^{\infty}\left[\frac{\operatorname{Res}_{\gamma_{z m}^{(3)}} f(w)}{\gamma_{z m}^{(3)}-\gamma_{z p}^{(1)}}+\frac{\operatorname{Res}_{\gamma_{z m}^{(3)}} f(w) R_{p}}{\gamma_{z m}^{(3)}+\gamma_{z p}^{(1)}}\right]+\left[\frac{\operatorname{Res}_{\frac{\omega}{i V}} f(w)}{\frac{\omega}{i V}-\gamma_{z p}^{(1)}}+\frac{\operatorname{Res}_{\frac{\omega}{i V}} f(w) R_{p}}{\frac{\omega}{i V}+\gamma_{z p}^{(1)}}\right]+f\left(\gamma_{z p}^{(1)}\right)+f\left(-\gamma_{z p}^{(1)}\right) R_{p}=0
$$




$$
\sum_{m=1}^{\infty}\left[\frac{\operatorname{Res}_{\gamma_{m}^{(3)}}^{(3)} f(w)}{\gamma_{z m}^{(3)}+\gamma_{z p}^{(1)}}+\frac{\operatorname{Res}_{\gamma_{m}^{(3)}}^{(3)} f(w) R_{p}}{\gamma_{z m}^{(3)}-\gamma_{z p}^{(1)}}\right]+\left[\frac{\operatorname{Res}_{\frac{\omega}{V}} f(w)}{\frac{\omega}{i V}+\gamma_{z p}^{(1)}}+\frac{\operatorname{Res}_{\frac{\omega}{V}} f(w) R_{p}}{\frac{\omega}{i V}-\gamma_{z p}^{(1)}}\right]+f\left(-\gamma_{z p}^{(1)}\right)+f\left(\gamma_{z p}^{(1)}\right) R_{p}=0
$$

$$
\begin{gathered}
\sum_{m=1}^{\infty} \frac{\operatorname{Res}_{\gamma_{z m}^{(3)}} f(w)}{\gamma_{z m}^{(3)}-\gamma_{z n}^{(2)}}+\frac{\operatorname{Res}_{i \bar{\omega}} f(w)}{\frac{\omega}{i V}-\gamma_{z n}^{(2)}}=0, \\
\sum_{m=1}^{\infty} \frac{\operatorname{Res}_{\gamma_{z m}^{(3)}}^{(3)} f(w)}{\gamma_{z m}^{(3)}+\gamma_{z n}^{(2)}}+\frac{\operatorname{Res}_{i V} f(w)}{\frac{\omega}{i V}+\gamma_{z n}^{(2)}}+f\left(-\gamma_{z n}^{(2)}\right)=0,
\end{gathered}
$$

Note that

$$
\operatorname{Res}_{\frac{\omega}{i V}} f(w)=P g\left(\frac{\omega}{i V}\right),
$$

where $g(w)$ is given by (34). Let us compare our systems (A10), (A11), (A18), and (A19) with relations (B4), (B5), (B6), and (B7), correspondingly. We put

$$
\operatorname{Res}_{\gamma_{z m}^{(3)}} f(w)=\tilde{A}_{m},
$$

and determine coefficient $P$ so that (iii) is fulfilled, i.e.

$$
P=\frac{i q}{2 c} \frac{i s_{0}^{2} h_{0}}{g\left(\frac{\omega}{i V}\right)} .
$$

At this step system (A18) is formally fulfilled. Next, we put

$$
\begin{aligned}
\tilde{C}_{n}= & f\left(-\gamma_{z n}^{(2)}\right)\left[2 \gamma_{z n}^{(2)}\right]^{-1}, \\
& -\frac{i q}{2 c b J_{1}\left(j_{0 p}\right)}\left[R_{p} F_{v p}^{-}+F_{v p}^{+}-R_{p} F_{d p}^{+}-F_{d p}^{-}\right] \\
& -\frac{4 \gamma_{z p}^{(1)} \kappa_{z p}^{(1)} \tilde{B}_{p}}{\kappa_{z p}^{(1)}+\varepsilon \gamma_{z p}^{(1)}}=f\left(-\gamma_{z p}^{(1)}\right)+f\left(\gamma_{z p}^{(1)}\right) R_{p},
\end{aligned}
$$

and systems (A19) and (A11) are formally fulfilled as well. Equation (26) follows from Eqs. (B12) and (A13), Eqs. (27) and (28) follow from (B11) and (A20). Finally, we put

$$
\begin{aligned}
& -\frac{i q}{2 c b J_{1}\left(j_{0 p}\right)}\left[F_{v p}^{-}+F_{v p}^{+} R_{p}-F_{d p}^{+}-F_{d p}^{-} R_{p}\right] \\
& \quad=f\left(\gamma_{z p}^{(1)}\right)+f\left(-\gamma_{z p}^{(1)}\right) R_{p}
\end{aligned}
$$

and the system (A10) is also fulfilled. Equation (B13) is the relation for determination of unknown zeros $\Gamma_{p}$ (38). After algebraic transformations it can be rewritten in the following form:

$$
\Delta_{p}\left(\left\{\Delta_{m}\right\}\right)=\frac{b}{\pi} \frac{G_{p} u_{p}\left[\Gamma_{p}-\omega /(i V)\right]-2 \gamma_{z p}^{(1)} R_{p}}{v_{p+}+R_{p} v_{p-}},
$$

where

$$
u_{p}\left(\left\{\Delta_{m}\right\}\right)=\left.\frac{g(w)}{1-\frac{w}{\Gamma_{p}}}\right|_{w=\frac{\omega}{i V}}
$$

$$
\begin{gathered}
v_{p \pm}\left(\left\{\Delta_{m}\right\}\right)=\left.\frac{f(w) / P}{1-\frac{w}{\Gamma_{p}}}\right|_{w= \pm \gamma_{z p}^{(1)}}, \\
G_{p}=\frac{F_{d p}^{+}+R_{p} F_{d p}^{-}-F_{v p}^{-}-R_{p} F_{v p}^{+}}{b J_{1}\left(j_{0 p}\right) s_{0}^{2} h_{0}} .
\end{gathered}
$$

Equation (B14) is complicated nonlinear system for $\Delta_{p}$ because expression in the right-hand side depends on all unknown $\left\{\Delta_{m}\right\}$ through $u_{p}$ and $v_{p \pm}$, this fact is underlined by the argument $\left\{\Delta_{m}\right\}$ of $\Delta_{p}, u_{p}$ and $v_{p \pm}$. This system can be solved numerically using iteration procedure. Possibility to control the convergence of this procedure is connected with Meixner edge condition (B2).

As it was shown in [21,23], condition (B2) dictates the following asymptotic behavior of coefficient $A_{p}$ for $p \rightarrow \infty$ :

$$
A_{p} \sim p^{-(1+\tau)}, \quad \tilde{A}_{p} \sim p^{-(1 / 2+\tau)} .
$$

This in turn results in the asymptotic behavior of $f(w)$ determined by condition (iv). Since asymptotic of $f(w)$ is determined by asymptotic of $\Gamma_{p}, \gamma_{z n}^{(2)}$ and $\gamma_{z m}^{(3)}$ for large numbers, the asymptotic of $\gamma_{z n}^{(2)}$ and $\gamma_{z m}^{(3)}$ can be easily learned from their definitions (24) and (23), $\Gamma_{p}$ should behave as follows for $p \rightarrow \infty$ :

$$
\Gamma_{p} \sim \frac{\pi}{b}(p-1 / 4+\tau), \quad \Delta_{p} \sim \tau .
$$

Therefore, the iteration process for solving (B14) is organized as follows. We fix quantity $N$ of $\Delta_{m}, m=$ $1,2, \ldots N$ to be found. For zero-order approximation, we put $\Delta_{m}=\tau$ for all $m$ in the right-hand side of (B14) and calculate first-order approximation for $\Delta_{p}, p=1,2, \ldots N$. Then we substitute these calculated $\left\{\Delta_{m}\right\}$ in the right-hand side of (B14) and calculate second-order approximation, etc. After these iterations have converged (relative difference in $\Delta_{N}$ for two consequent steps is within the accuracy), we compare $\Delta_{N}$ with $\tau$ : if $\Delta_{N} \approx \tau$ within 
accepted accuracy, process is stopped, otherwise $N$ and/or accuracy of calculations is changed and procedure repeats.

\section{APPENDIX C: FREQUENCY SPECTRUM OF THE SCATTERED FIELD}

According to (17), (18), and (19), spectrum of the scattered field is determined by spectrum of coefficients $\left\{A_{m}\right\},\left\{B_{m}\right\}$ and $\left\{C_{n}\right\}$. Here we present analytical proving that real spectrum of these coefficients contains the same Cherenkov poles as the incident field in the inner dielectric waveguide. For example, let us consider coefficient $\tilde{A}_{p}$. In accordance with (25) or (B9), we obtain:

$$
\tilde{A}_{p}=P \frac{\left(\gamma_{z p}^{(3)}-\gamma_{z 0}^{(2)}\right) \prod_{n=1}^{\infty}\left(1-\frac{\gamma_{z p}^{(3)}}{\gamma_{z n}^{(2)}}\right) \prod_{s=1}^{\infty}\left(1-\frac{\gamma_{z p}^{(3)}}{\Gamma_{s}}\right)}{\left(\frac{\frac{\omega}{i V}}{\gamma_{z p}^{(3)}}-1\right) \prod_{\substack{m=1 \\ m \neq p}}^{\infty}\left(1-\frac{\gamma_{z p}^{(3)}}{\gamma_{z m}^{(3)}}\right)} Q\left(\gamma_{z p}^{(3)}\right) .
$$

None of the terms in denominator can be zero for real frequencies, therefore only coefficient $P$ can have poles. Definition of $P(36)$ or (B10) can be rewritten as follows:

$$
P=\frac{-q s_{0}^{2} h_{0} \prod_{m=1}^{\infty}\left(1-\frac{\frac{\omega}{i V}}{\gamma_{z m}^{(3)}}\right) Q\left(-\frac{\omega}{i V}\right)}{2 c\left(\frac{\omega}{i V}-\gamma_{z 0}^{(2)}\right) \prod_{n=1}^{\infty}\left(1-\frac{\frac{\omega}{i V}}{\gamma_{z n}^{(2)}}\right) \prod_{s=1}^{\infty}\left(1-\frac{\frac{\omega}{i V}}{\Gamma_{s}}\right)} .
$$

In the denominator, the first term does not equal zero for real $\omega$ and $\beta \neq 1$, the first product does not equal zero for real $\omega$ as well because $\omega /(i V) \neq \gamma_{z n}^{(2)}$, therefore only the second product is a candidate to have real zeros responsible for poles of $P$. As our numerical results indicate, zeros $\Gamma_{s}$ are specifically shifted in the complex plane so that Eq. (44) is fulfilled with high accuracy. Therefore, coefficient $P$ has poles for Cherenkov frequencies (14). Since $\left\{A_{m}\right\},\left\{B_{m}\right\}$ and $\left\{C_{n}\right\}$ are all proportional to $P$, the scattered field spectrum contains the same Cherenkov poles $\omega_{l}^{\mathrm{Ch}}$ as the incident field in the area 1, which has to be proved.

[1] G. P. Williams, Filling the THz gap-high power sources and applications, Rep. Prog. Phys. 69, 301 (2006).

[2] M. I. Bakunov, R. V. Mikhaylovskiy, S. B. Bodrov, and B.S. Luk'yanchuk, Reversed Cherenkov emission of terahertz waves from an ultrashort laser pulse in a sandwich structure with nonlinear core and left-handed cladding, Opt. Express 18, 1684 (2010).

[3] H. Wen, K.-J. Kim, A. Zholents, J. Byrd, and A. Cavalleri, Preface to Special Topic: Intense terahertz sources for timeresolved studies of matter, Rev. Sci. Instrum. 84, 022501 (2013).

[4] D. Kazakov, M. Piccardo, Y. Wang, P. Chevalier, T. S. Mansuripur, F. Xie, C.-e. Zah, K. Lascola, A. Belyanin, and F. Capasso, Self-starting harmonic frequency comb generation in a quantum cascade laser, Nat. Photonics 11, 789 (2017).

[5] B. D. O'Shea, G. Andonian, S. Barber, K. Fitzmorris, S. Hakimi, J. Harrison, P. D. Hoang, M. J. Hogan, B. Naranjo, O. B. Williams, V. Yakimenko, and J. Rosenzweig, Observation of acceleration and deceleration in gigaelectron-volt-per-metre gradient dielectric wakefield accelerators, Nat. Commun. 7, 12763 (2016).

[6] S. Antipov, M. Babzien, C. Jing, M. Fedurin, W. Gai, A. Kanareykin, K. Kusche, V. Yakimenko, and A. Zholents, Subpicosecond Bunch Train Production for a Tunable mJ Level THz Source, Phys. Rev. Lett. 111, 134802 (2013).

[7] S. Antipov, C. Jing, S. Baryshev, A. Kanareykin, D. Wang, W. Gai, A. Zholents, and M. Fedurin, THz Radiation generation in a multimode wakefield structure, in Proceedings of the 6th International Particle Accelerator Conference (IPAC2015) (2015), pp. 1929-1931, DOI: 10.18429/JACoW-IPAC2015-TUPMA042.

[8] S. N. Galyamin, A. V. Tyukhtin, S. Antipov, and S. S. Baturin, Terahertz radiation from an ultra-relativistic charge exiting the open end of a waveguide with a dielectric layer, Opt. Express 22, 8902 (2014).

[9] S. Antipov, S. Baryshev, R. Kostin, S. Baturin, J. Qiu, C. Jing, C. Swinson, M. Fedurin, and D. Wang, Efficient extraction of high power $\mathrm{THz}$ radiation generated by an ultra-relativistic electron beam in a dielectric loaded waveguide, Appl. Phys. Lett. 109, 142901 (2016).

[10] D. Wang, X. Su, L. Yan, Y. Du, Q. Tian, Y. Liang, L. Niu, W. Huang, W. Gai, C. Tang, and S. Antipov, Phase control with two-beam interferometry method in a terahertz dielectric wakefield accelerator, Appl. Phys. Lett. 111, 174102 (2017).

[11] L. A. Vainshtein, The Theory of Diffraction and the Factorization Method (Golem, Boulder, Colorado, 1969).

[12] R. Mittra and S. Lee, Analytical Techniques in the Theory of Guided Waves (MacMillan, London, 1971).

[13] B. M. Bolotovskii and E. A. Galstyan, Diffraction and diffraction radiation, Usp. Fiz. Nauk 170, 809 (2000).

[14] S. Kheifets and S. Heifets, Radiation of a charge in a perfectly conducting cylindrical pipe with a jump in its cross section, Report No. SLAC-PUB-3965, 1986.

[15] S. Kheifets, L. Palumbo, and V. G. Vaccaro, Electromagnetic fields scattered by a charge moving on the axis of a semi-infinite circular waveguide: Radiation spectrum and longitudinal impedance, IEEE Trans. Nucl. Sci. 34, 1094 (1987).

[16] L. Palumbo, Analytical calculation of the impedance of a discontinuity, Part. Accel. 25, 201 (1990).

[17] A. V. Tyukhtin, Self-acceleration of a charge traveling into a waveguide, Phys. Rev. ST Accel. Beams 17, 021303 (2014).

[18] M. Ivanyan, A. Grigoryan, A. Tsakanian, and V. Tsakanov, Wakefield radiation from the open end of an internally coated metallic tube, Phys. Rev. ST Accel. Beams 17, 074701 (2014).

[19] G. F. VanBlaricum and R. Mittra, A modified residuecalculus technique for solving a class of boundary value problems part I: Waveguide discontinuities, IEEE Trans. Microwave Theory Tech. 17, 302 (1969). 
[20] G. Voskresenskii and S. Zhurav, Radiation of magnetic waves from the open end of a flat waveguide partially filled with dielectric, Radiotekhnika i Electronika 23, 2505 (1978).

[21] S. N. Galyamin, A. V. Tyukhtin, A. M. Altmark, and S.S. Baturin, Radiation of a bunch flying from the open end of a waveguide with a dielectric loading, in Proceedings of the 25th Russian Particle Accelerator Conference (RuPAC2016) (2016), pp. 518-520.

[22] S. N. Galyamin, V. V. Vorobev, A. M. Altmark, A. A. Grigoreva, A. V. Tyukhtin, and S. Antipov, Radiation of a charge exiting open-ended waveguide with dielectric filling, in 2017 Progress In Electromagnetics Research Symposium-Spring (PIERS) (2017) pp. 1083-1088, DOI: 10.1109/PIERS.2017.8261907.

[23] S. N. Galyamin, A. V. Tyukhtin, and V. V. Vorobev, Radiation from open ended waveguide with dielectric loading, Nucl. Instrum. Methods Phys. Res., Sect. B 402, 144 (2017).

[24] S. N. Galyamin, A. V. Tyukhtin, V. V. Vorobev, A. A. Grigoreva, and A. Aryshev, Bunch imaging at the open end of an embedded circular waveguide, IEEE Trans. Microwave Theory Tech. 66, 2100 (2018).

[25] S. Galyamin, A. Tyukhtin, V. Vorobev, and A. Aryshev, Bunch radiation from a semi-infinite waveguide with dielectric filling inside a waveguide with larger radius, J. Instrum. 13, C02012 (2018).

[26] R. L. Gluckstern, Coupling impedance of a single hole in a thick-wall beam pipe, Phys. Rev. A 46, 1106 (1992).

[27] R. L. Gluckstern, Coupling impedance of many holes in a liner within a beam pipe, Phys. Rev. A 46, 1110 (1992).

[28] G. V. Stupakov and S. S. Kurennoy, Trapped electromagnetic modes in a waveguide with a small discontinuity, Phys. Rev. E 49, 794 (1994).

[29] S. S. Kurennoy, Trapped modes in waveguides with many small discontinuities, Phys. Rev. E 51, 2498 (1995).

[30] S. S. Kurennoy, R. L. Gluckstern, and G. V. Stupakov, Coupling impedances of small discontinuities: A general approach, Phys. Rev. E 52, 4354 (1995).

[31] A. V. Fedotov and R. L. Gluckstern, Frequency dependence of the penetration of electromagnetic fields through an elliptical hole in a thin metallic wall, Phys. Rev. E 54, 1930 (1996).

[32] A. V. Fedotov and R. L. Gluckstern, Analytic and numerical analysis of the longitudinal coupling impedance of a rectangular slot in a thin coaxial liner, Phys. Rev. E 56, 3583 (1997).

[33] A. V. Fedotov and R. L. Gluckstern, Impedance and resonance issues for a long rectangular slot in a coaxial liner, Phys. Rev. E 56, 7217 (1997).

[34] R. L. Gluckstern and S.S. Kurennoy, Impedances of azimuthally symmetric irises and cavities with semielliptical profile in a beam pipe, Phys. Rev. E 55, 3533 (1997).

[35] A. V. Fedotov and R. L. Gluckstern, Longitudinal coupling impedance of a small hole in a coaxial liner near the cutoff frequencies, Phys. Rev. ST Accel. Beams 1, 024401 (1998).

[36] S. De Santis, M. Migliorati, L. Palumbo, and M. Zobov, Coupling impedance of a hole in a coaxial beam pipe, Phys. Rev. E 54, 800 (1996).
[37] S. De Santis and L. Palumbo, Impedance of a coaxial cavity coupled to the beam pipe through a small hole, Phys. Rev. E 55, 2052 (1997).

[38] S. De Santis, A. Mostacci, and L. Palumbo, Interference effects on the coupling impedance of many holes in a coaxial beam pipe, Phys. Rev. E 56, 5990 (1997).

[39] S. De Santis, A. Mostacci, L. Palumbo, and B. Spataro, Analytical expressions for the coupling impedance of a long narrow slot in a coaxial beam pipe, Phys. Rev. E 58, 6565 (1998).

[40] S. Petracca, Beam coupling impedances for perforated beam pipes with general shape from impedance boundary conditions, Phys. Rev. E 60, 6030 (1999).

[41] R. Gluckstern and B. Zotter, Analysis of shielding charged particle beams by thin conductors Phys. Rev. ST Accel. Beams 4, 024402 (2001).

[42] S. S. Kurennoy, Coupling impedances of small discontinuities: Dependence on beam velocity, Phys. Rev. ST Accel. Beams 9, 054201 (2006).

[43] D. Alesini, M. Di Giosa, A. Mostacci, and L. Palumbo, Conceptual study of an ultra-short bunch length monitor, in Proceedings of the 19th Particle Accelerator Conference, Chicago, IL, 2001 (IEEE, Piscataway, NJ, 2001), pp. 2359-2361.

[44] R. Bossart, High precision beam position monitor using a re-entrant coaxial cavity, in Proceedings of the 17th International Linear Accelerator Conference (LINAC1994), Tsukuba, Japan, 1994 (KEK, Tsukuba, Japan, 1994), pp. 851-853.

[45] C. Simon, M. Luong, S. Chel, O. Napoly, J. Novo, D. Roudier, N. Rouvière, N. Baboi, N. Mildner, and D. Nölle, Performance of a reentrant cavity beam position monitor, Phys. Rev. ST Accel. Beams 11, 082802 (2008).

[46] A. Novokhatski, F.-J. Decker, H. Smith, and M. Sullivan, Wakefields in SLAC linac collimators, Phys. Rev. ST Accel. Beams 17, 124401 (2014).

[47] A. Dexter, G. Burt, and R. Apsimon, Active lower order mode damping for the four rod LHC crab cavity, Nucl. Instrum. Methods Phys. Res., Sect. A 844, 62 (2017).

[48] B. Hall, G. Burt, R. Apsimon, C. J. Lingwood, A. Tutte, A. Grudiev, A. Macpherson, M. Navarro-Tapia, R. Calaga, K. G. Hernández-Chahín, R. B. Appleby, and P. Goudket, Design and testing of a four rod crab cavity for High Luminosity LHC, Phys. Rev. Accel. Beams 20, 012001 (2017).

[49] N. Biancacci, V. G. Vaccaro, E. Métral, B. Salvant, M. Migliorati, and L. Palumbo, Impedance studies of 2D azimuthally symmetric devices of finite length, Phys. Rev. ST Accel. Beams 17, 021001 (2014).

[50] G. N. Afanasiev, Vavilov-Cherenkov and Synchrotron Radiation: Foundations and Applications (Springer, New York, 2004).

[51] A. V. Tyukhtin and S. N. Galyamin, Vavilov-Cherenkov radiation in passive and active media with complex resonant dispersion, Phys. Rev. E 77, 066606 (2008).

[52] S. N. Galyamin and A. V. Tyukhtin, Electromagnetic field of a moving charge in the presence of a left-handed medium, Phys. Rev. B 81, 235134 (2010).

[53] B. M. Bolotovskii, Theory of the Vavilov-Cherenkov effect (III), Physics-Uspekhi 4, 781 (1962). 
[54] G. M. Garibian, Contribution to the theory of transition radiation, Sov. Phys. JETP 6, 1079 (1958).

[55] S. N. Galyamin, A. V. Tyukhtin, A. Kanareykin, and P. Schoessow, Reversed Cherenkov-Transition Radiation by a Charge Crossing a Left-Handed Medium Boundary, Phys. Rev. Lett. 103, 194802 (2009).
[56] S. N. Galyamin and A. V. Tyukhtin, Electromagnetic field of a charge traveling into an anisotropic medium, Phys. Rev. E 84, 056608 (2011).

[57] A. P. Prudnikov, Y. A. Brychkov, and O. I. Marichev, Integrals and Series, Elementary Functions Vol. 1 (Gordon \& Breach Science Publishers, New York, 1986). 